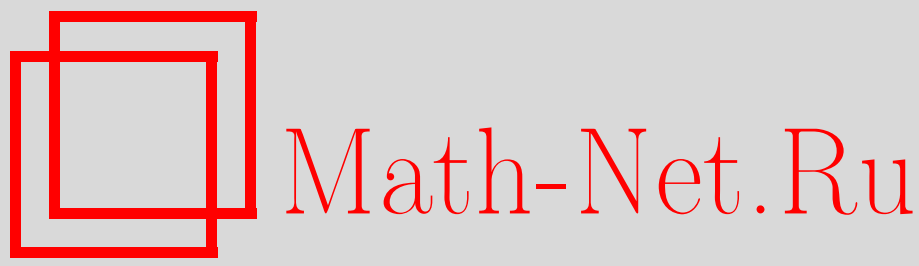

М. Э. Казарян, С. К. Ландо, К теории пересечений на пространствах Гурвица, Изв. РАН. Сер. матем., 2004, том 68, выпуск 5, 91-122

DOI: https://doi.org/10.4213/im504

Использование Общероссийского математического портала Math-Net.Ru подразумевает, что вы прочитали и согласны с пользовательским соглашением

http://www . mathnet.ru/rus/agreement

Параметры загрузки:

IP: 54.81 .137 .203

26 апреля 2023 г., $16: 48: 52$ 


\title{
К теории пересечений на пространствах Гурвица
}

\begin{abstract}
Пространства модулей алгебраических кривых и тесно связанные с ними пространства Гурвица - пространства мероморфных функций на кривых - появляются естественным образом во многих задачах алгебраической геометрии и математической физики, особенно в связи с теорией струн и теорией инвариантов Громова-Виттена. К изучению геометрии и топологии этих пространств сводится, в частности, классическая задача Гурвица о подсчете количества топологически различных разветвленных накрытий над сферой с предписанными типами ветвлений. Кольца когомологий этих пространств довольно сложны даже в простейшем случае рациональных кривых и функций. Тем не менее, наиболее важные с точки зрения приложений когомологические классы, двойственные по Пуанкаре стратам функций с фиксированными особенностями, выражаются в терминах относительно простого набора "основных" (в некотором смысле, тавтологических) классов. Цель статьи - выделить эти основные классы, описать соотношения между ними и найти выражения для стратов в терминах этих классов. Наш подход основан на теории Тома универсальных многочленов особенностей, которая распространена на случай мультиособенностей в работах первого автора. Хотя задача Гурвица в полном объеме все еше не решена, данный подход позволяет существенно продвинуться в ее решении, а также в понимании геометрии и топологии пространств Гурвица.

Библиография: 22 наименования.
\end{abstract}

\section{§1. Введение}

1.1. Задача Гурвица. В статье [8] А. Гурвиц поставил следующую задачу: подсчитать количество классов изоморфизма разветвленных накрытий двумерной сферы поверхностью рода $g$ с предписанными точками ветвления в сфере-образе и предписанным типом ветвления над каждой точкой ветвления. (Точную формулировку задачи мы приводим ниже.) В течение прошлого века эта задача неоднократно привлекала внимание ученых, однако это направление исследований стало в математике одним из центральных лишь недавно. Взрыв интереса к нему объясняется прежде всего приложениями, которые задача Гурвища нашла в современной математической физике, а именно в теории струн и теории инвариантов Громова-Виттена.

Вплоть до сегодняшнего дня задача Гурвица не имеет адекватного решения: известные к настояшему моменту формулы, выражаюшие числа накрытий в терминах данных ветвления, приводят к прозрачным ответам лишь в некоторых частных случаях. В [1], [2] обнаружена связь между разветвленными накрытиями сферы

Работа выполнена при финансовой поддержке РФФИ (первого автора - грантом № 04-0100762, второго автора - грантом № 02-01-22004). 
и теорией пересечений на пространствах модулей функций, определенных на алгебраических кривых - пространствах Гурвица. Наличие этой связи возрождает надежды на получение простых общих ответов. В статьях [1], [2] задача Гурвища фактически сведена к некоторой задаче о геометрии пространств модулей, которая представляет и независимый интерес. В силу конструкции из [2] она связана со ставшей уже классической задачей о геометрии пространств модулей алгебраических кривых.

В настоящей статье мы анализируем ту часть геометрии пространств Гурвица, которая относится к задаче Гурвища.

1.2. Пространства Гурвица и когомологические классы стратов. Рассмотрим разветвленные накрытия двумерной сфиеры поверхностями рода $g$; чеpeз $n$ обозначим степень накрытия. Помимо топологических накрытий мы также рассмотрим мероморфные функции степени $n$ на алгебраических кривых рода $g$. Разумеется, с топологической точки зрения каждая такая функция является разветвленным накрытием. Обозначим через $\mathcal{H}_{g, n}$ пространство таких функций, обладаюших следуюшими свойствами:

- все полюсы функций простые, т. е. мероморфная функция имеет $n$ полюсов порядка один; кроме того, мы предполагаем, что все полюсы каждой функции занумерованы;

- сумма критических значений функции равна нулю.

Согласно [2] это пространство является гладким комплексным орбиобразием (или даже гладким комплексным многообразием, если $g=0$ или $n$ достаточно велико). Оно расслоено над пространством модулей $\mathcal{M}_{g, n}$ комплексных кривых рода $g$ с $n$ отмеченными точками: всякой мероморфной функции можно сопоставить кривую ее определения с $n$ отмеченными полюсами.

Пространство $\mathcal{H}_{g, n}$ имеет пополнение, обозначаемое через $\overline{\mathcal{H}}_{g, n}$ и состоящее из стабильных мероморфных функций [2], [14]. Его граница $\overline{\mathcal{H}}_{g, n} \backslash \mathcal{H}_{g, n}$ состоит из стабильных функций, кривая определения которых особа, причем единственные допустимые особенности - это узлы (точки простого двойного самопересечения). Проекция $\mathcal{H}_{g, n} \rightarrow \mathcal{M}_{g, n}$ продолжается до проекции $\overline{\mathcal{H}}_{g, n} \rightarrow \overline{\mathcal{M}}_{g, n}$ пополненного пространства в пространство модулей стабильных кривых с отмеченными точками. Слои этой проекции - векторные пространства, поскольку линейная комбинация мероморфных функций с полюсами не выше первого порядка в отмеченных точках является функцией того же вида. Отметим, однако, что эта проекция не является векторным расслоением, поскольку размерность слоя может меняться от одной точки базы к другой. Послойная проективизация $P \overline{\mathcal{H}}_{g, n}$ пополненного пространства Гурвица является компактным комплексным орбиобразием. Оно и будет служить нашим основным пространством модулей, а все остальные нужные пространства будут конструироваться на его основе. Допуская некоторую вольность речи, мы говорим ниже о многообразиях и подмногообразиях, имея в виду, что наши пространства являются на самом деле орбиобразиями и подорбиобразиями (т.е. устроены локально как факторпространства комплексного шара по модулю действия конечной группы). 
На пространстве $P \overline{\mathcal{H}}_{g, n}$, поскольку оно является проективизацией, выделен естественный класс вторых когомологий, а именно первый класс Черна тавтологического пучка, который мы обозначаем через $\psi=\psi_{g, n}=c_{1}(\mathcal{O}(1)) \in H^{2}\left(P \overline{\mathcal{H}}_{g, n}\right)$.

$\mathrm{C}$ другой стороны, в этом пространстве есть подмногообразия, состоящие из функций с вырожденными ветвлениями. По формуле Римана-Гурвища общая мероморфная функция степени $n$ на кривой рода $g$ имеет $2 n+2 g-2$ точки невырожденного ветвления (как в образе, так и в прообразе). Функции с меньшим количеством точек ветвления в сфере-образе образуют дискриминант в пространстве функций. Каждой точке ветвления в образе можно сопоставить разбиение числа $n$, представляющее собой (неупорядоченный) набор кратностей прообразов данной точки. Нам будет удобнее, однако, пользоваться приведенными разбиениями , т. е. наборами уменьшенных на 1 кратностей прообразов точек ветвления. Замыкание в $P \overline{\mathcal{H}}_{g, n}$ множества функций, имеюших ветвления предписанного типа, будем обозначать через $\sigma_{\alpha_{1}, \ldots, \alpha_{c}}$, где индекс состоит из приведенных разбиений над точками вырожденного ветвления. Эти подмногообразия называются $\mathrm{cmpa-}$ тами дискриминанта. Так, $\sigma_{2^{1}} \subset P \overline{\mathcal{H}}_{g, n}$ обозначает каустику, т. е. страт, состоящий из функций, две точки ветвления которых в прообразе склеились, а $\sigma_{1^{2}}-$ страт Максвелла, открытая часть которого состоит из функций, принимаюших одинаковые значения в двух различных критических точках. Каустика и страт Максвелла - единственные страты (комплексной) коразмерности 1. Аналогично, страт $\sigma_{2^{1}, 1^{2}}$, имеюший коразмерность 2, представляет собой замыкание множества функций с двумя вырожденными критическими значениями, над одним из которых имеется точка ветвления кратности 3 , а над другим - две простые точки ветвления, и т. д. Число невырожденных критических точек (соответствующих приведенному разбиению $1^{1}$ ) для общей функции данного страта легко вычисляется по формуле Римана-Гурвица. Для упрощения обозначений мы не включаем эти невырожденные приведенные разбиения, а также значения $g$ и $n$ в обозначения стратов.

Каждый страт представляет собой комплексное подмногообразие чистой размерности в $P \overline{\mathcal{H}}_{g, n}$, а значит, определяет по двойственности Пуанкаре однородньй элемент кольца когомологий

$$
H^{*}\left(P \overline{\mathcal{H}}_{g, n}\right)=H^{*}\left(P \overline{\mathcal{H}}_{g, n}, \mathbb{Q}\right)
$$

(Всюду далее нас интересуют когомологии с рациональными коэффициентами, и мы опускаем это основное кольцо в обозначениях когомологий.) Степень этого элемента совпадает с (вещественной) коразмерностью страта. Его произведение с дополнительной степенью класса $\psi$ есть просто рациональное число, которое и это результат геометрического подхода, предложенного в [2], - тесно связано с интересуюшим нас числом накрытий. Прежде чем описывать эту связь, приведем строгую формулировку задачи Гурвища.

Два разветвленных накрытия $f_{1}: C_{1} \rightarrow S^{2}, f_{2}: C_{2} \rightarrow S^{2}$ двумерной сферы называются изоморфными, если сушествует гомеоморфизм $h: C_{1} \rightarrow C_{2}$ такой, что $f_{1}=f_{2} \circ h$. Ясно, что у изоморфных разветвленных накрытий точки ветвления в сфере-образе и типы ветвления над этими точками совпадают так же, как и их степени $n_{1}, n_{2}$ и рода $g_{1}, g_{2}$ накрывающих кривых, $n_{1}=n_{2}=n, g_{1}=g_{2}=g$. 
Точка ветвления в сфере-образе называется невырожденной, или простой, если она имеет $n-1$ геометрически различных прообразов, один из которых является точкой ветвления кратности 2 , а остальные $n-2$ - точки гладкости накрытия. Как упоминалось выше, мы сопоставляем каждой точке ветвления в образе приведенное разбиение $\alpha$, состоящее из уменьшенных на единицу кратностей ее особых прообразов. Множество всех разбиений $\alpha$ для данного разветвленного накрытия назьвается данными ветвления. Определим число Гурвица $h_{\alpha_{1}, \alpha_{2}, \ldots}$ следующим равенством:

$$
h_{\alpha_{1}, \alpha_{2}, \ldots}=\sum_{f} \frac{1}{|\operatorname{Aut}(f)|}
$$

где суммирование ведется по всем классам изоморфизма разветвленных накрытий $f$ сферы степени $n$ поверхностью рода $g$ с приведенными разбиениями $\alpha_{1}, \alpha_{2}, \ldots$ над вырожденными точками ветвления, а через $|\operatorname{Aut}(f)|$ обозначено число элементов в группе автоморфизмов такого разветвленного накрытия (такая группа всегда конечна). Разумеется, числа Гурвица зависят от $g$ и $n$, но мы не включаем эти параметры в обозначения.

Задача Гурвица состоит в подсчете чисел Гурвица, и с геометрией пространств Гурвица ее связывает следующая теорема, которая для случая $g=0$ была явно сформулирована в [16] и которая непосредственно вытекает из результатов [2].

Теорема 1.1. Имеет место равенство

$$
h_{\alpha_{1}, \alpha_{2}, \ldots}=\frac{\left|\operatorname{Aut}\left(\alpha_{1}, \alpha_{2}, \ldots\right)\right|}{n !}\left\langle\sigma_{\left.\alpha_{1}, \alpha_{2}, \ldots, \psi^{d}\right\rangle}\right.
$$

где через $\left|\operatorname{Aut}\left(\alpha_{1}, \alpha_{2}, \ldots\right)\right|$ обозначен порядок группы автоморфизмов множества приведенных разбиений, т.е. произведение факториалов чисел совпадающих разбиений, а угловые скобки обозначают умножение в когомологиях пространства $P \overline{\mathcal{H}}_{g, n}$.

Теорема 1.1 сводит задачу Гурвица к некоторому вопросу о когомологиях пространств Гурвица. Несмотря на видимое усложнение, вызванное заменой комбинаторно-групповой задачи алгебро-геометрической, эта геометрическая интерпретация не только проясняет известные вычислительные результаты (см. [8], [6], $[15],[2])$, но и приводит к новым (см., например, [16]).

Вообще говоря, кольца когомологий пространств модулей стабильных кривых и тесно связанных с ними пространств Гурвица очень сложны даже в случае рациональных кривых и функций $(g=0)$. Опыт авторов подсказывает, однако, что все естественные геометрические классы, связанные с исчислением особенностей (а также мультиособенностей и мультимультиособенностей - это в точности классы, играющие центральную роль в задаче Гурвица), выражаются в терминах относительно простого набора "основных" (в некотором смысле тавтологических) классов. Наша цель - выделить эти основные классы, описать соотношения между ними и найти выражения для стратов в терминах этих классов. 
1.3. Описание основных результатов. Начнем описание с результатов о числах Гурвища.

ТЕОРема 1.2. Для рачиональных $($ т. е. относящихся $\kappa$ случаю $g=0)$ чисел Гурвица выполнены следующие формуль:

$$
\begin{aligned}
& h_{2^{1}, 2^{1}}=\frac{3}{4}\left(27 n^{2}-137 n+180\right) \frac{(2 n-6) !}{(n-3) !} n^{n-6}, \\
& h_{2^{1}, 1^{2}}=3\left(3 n^{2}-15 n+20\right) \frac{(2 n-6) !}{(n-4) !} n^{n-6}, \\
& h_{1^{2}, 1^{2}}=2\left(2 n^{3}-16 n^{2}+43 n-40\right) \frac{(2 n-6) !}{(n-4) !} n^{n-6} .
\end{aligned}
$$

Эти формулы относятся к случаю мультимультиособенностей, т.е. к ситуации, в которой ветвление невырождено над несколькими точками в сфере-образе. Для мультиособенностей, где ветвление вырождено лишь над одной точкой, рациональные числа Гурвица вычисляются по хорошо известной формуле Гурвица. Первая формула в теореме 1.2 получена Д. Звонкиным в [21], две другие, насколько нам известно, являются новыми.

Для доказательства этих результатов мы выводим некоторые соотношения в когомологиях пространств Гурвища на классы, представленные стратами дискриминанта, и класс $\psi$. Мы также будем активно использовать класс $\delta \in H^{2}\left(P \overline{\mathcal{H}}_{g, n}\right)$, представленный функциями на особых кривых. Класс $\delta$ очень близок к поднятию граничного классав в $\overline{\mathcal{M}}_{g, n}$ относительно проекции $\overline{\mathcal{H}}_{g, n} \rightarrow \overline{\mathcal{M}}_{g, n}$ (они различаются на дивизор функций, имеющих один полюс на некоторой рациональной неприводимой компоненте, прикрепленной к другим компонентам в единственной точке). Значение последнего в геометрии пространств модулей стабильных кривых хорошо известно. Например, индекс максимального самопересечения этого класса представляет собой оббем Вейля-Петерссона пространства модулей (см. [17]). Принципиальное отличие пространств Гурвица от пространств модулей кривых состоит в появлении еще одного естественного класса вторых когомологий, а именно класса $\psi$.

Отметим, что все приводимые в статье результаты справедливы не только в кольце когомологий, но и в кольце Чжоу пространства $P \overline{\mathcal{H}}_{g, n}$. Единственная причина, по которой мы говорим именно о когомологиях, состоит в том, что когомологические вычисления достаточны для предполагаемых вычислительных приложений.

Насколько нам известно, первые соотношения на страты в кольцах когомологий пространств Гурвища были получены в [16]. Они выражают каустику и страт Максвелла в пространствах рациональных функций $(g=0)$ через граничный страт и класс $\psi$ :

$$
\begin{aligned}
& \sigma_{2^{1}}=6(n-1) \psi-3 \delta, \\
& \sigma_{1^{2}}=2(n-1)(n-6) \psi+4 \delta .
\end{aligned}
$$

Ниже мы приводим новый вывод этих формул. Классы, комплексная коразмерность которых превьшает единицу, также подчиняются многочисленным соотношениям, которые более подробно описаны в основном тексте статьи, поскольку их описание требует дополнительных определений. 
1.4. Степень. Теорема 1.1 сводит вычисление числа Гурвища к вычислению индекса пересечения страта с дополнительной степенью класса $\psi$. Такой класс $\psi$ естественно возникает на любом пространстве орбит действия группы $\mathbb{C}^{*}$; он ведет себя функториально при эквивариантных отображениях многообразий, наделенных действием этой групшы. Именно поэтому индекс пересечения подмногообразия с дополнительной степенью этого класса естественно называть степенью.

Пусть $A$ - комплексное многообразие, и предположим, что мультипликативная группа $\mathbb{C}^{*}$ ненулевых комплексных чисел действует на нем без неподвижных точек. Пусть $B$ - многообразие орбит действия группы $\mathbb{C}^{*}$ на $A$, и предположим, что $B$ компактно. Обозначим через $\Psi=c_{1}(O(1)) \in H^{2}(B)$ тавтологический класс этого действия. Для произвольного класса $\beta \in H^{*}(B)$ назовем его степенью $\operatorname{deg} \beta$ результат спаривания:

$$
\operatorname{deg} \beta=\int_{B} \frac{\beta}{1-\Psi}=\int_{B} \beta\left(1+\Psi+\Psi^{2}+\Psi^{3}+\ldots\right) .
$$

Другими словами, степень однородного класса $\beta$ - это индекс его пересечения с классом $\Psi$, взятым в степени, равной коразмерности класса $\beta$.

ПримеР 1.3. Взвешенное проективное пространство $\mathbb{C} P_{w_{0}, \ldots, w_{n}}-$ это факторпространство дополнения к началу координат в векторном пространстве $\mathbb{C}^{n+1}$, на котором группа $\mathbb{C}^{*}$ действует следующим образом:

$$
\lambda:\left(x_{0}, \ldots, x_{n}\right) \mapsto\left(\lambda^{w_{0}} x_{0}, \ldots, \lambda^{w_{n}} x_{n}\right), \quad \lambda \in \mathbb{C}^{*}
$$

Целые показатели $w_{i}$ называются весами действия. Всякое непрерывное линейное представление группы $\mathbb{C}^{*}$ имеет такой вид. Степень взвешенного проективного пространства $\mathbb{C} P_{w_{0}, \ldots, w_{n}}$ с весами $w_{0}, \ldots, w_{n}$ равна $\left(\prod w_{i}\right)^{-1}$.

Из определений немедленно вытекает функториальность степени.

ПРЕДЛОЖЕНИЕ 1.4. Пусть группа $\mathbb{C}^{*}$ действует без неподвижньх точек на комплексных многообразиях $A_{1}, A_{2}$ одинаковой размерности, причем соответствующие пространства орбит $B_{1}, B_{2}$ компактны. Тогда степень әквивариантного отображения $f: A_{1} \rightarrow A_{2}$ не зависит от $f$ и определяется из равенства

$$
\operatorname{deg} B_{1}=\operatorname{deg} f \cdot \operatorname{deg} B_{2}
$$

Теорема 1.1 немедленно вытекает из этого предложения, если его применить к отображению $L_{g, n}: \overline{\mathcal{H}}_{g, n} \rightarrow \mathbb{C}^{2 g+2 n-3}$, переводящему мероморфную функцию в неупорядоченный набор ее критических значений (напомним, что согласно нашей договоренности сумма критических значений равна нулю).

1.5. Особенности, относительные классы Черна и универсальные многочлены. Равенства (1.1) получены в [16] путем подсчета индексов пересечений классов $\sigma_{2^{1}}, \sigma_{1^{2}}$ с базисным набором классов дополнительной размерности (т.е. комплексной размерности один, т. е. кривых). Однако при росте коразмерности стратов этот способ становится весьма трудоемким: как выбор базисного набора классов дополнительной размерности, так и подсчет индекса пересечения 
с таким классом - трудные задачи, подходы к эффективному решению которых отсутствуют. В настоящей работе мы предлагаем новый подход к поиску соотношений между классами когомологий. Этот подход основан на теории Тома универсальных многочленов особенностей [20], которая распространена на случай мультиособенностей в [10]-[13]. Объясним кратко суть этой теории.

Пусть $F: M \rightarrow N$ - общее голоморфиное отображение компактных комплексных многообразий (мы предполагаем для определенности, что размерность многообразий одинакова: хотя теория работает и для произвольных размерностей, мы будем применять ее лишь в случае совпадаюших размерностей). Полным классом Черна отображения $F$ называется отношение

$$
\begin{aligned}
c(F)= & \frac{c\left(F^{*} T N\right)}{c(T M)}=1+\left(c_{1}\left(F^{*}(T N)\right)-c_{1}(T M)\right)+\left(c_{2}\left(F^{*} T N\right)\right. \\
& \left.-c_{1}\left(F^{*} T N\right) c_{1}(T M)-c_{2}(T M)+c_{1}^{2}(T M)\right)+\ldots \in H^{*}(M)
\end{aligned}
$$

здесь функция $c(\cdot)$, аргументом которой является векторное расслоение, обозначает, как обычно, полный класс Черна этого расслоения. Однородные компоненты полного класса Черна называются классами Черна отображения $F$ и обозначаются $c_{i}(F) \in H^{2 i}(M)$ :

$$
\begin{aligned}
& c_{1}(F)=c_{1}\left(F^{*} T N\right)-c_{1}(T M), \\
& c_{2}(F)=c_{2}\left(F^{*} T N\right)-c_{1}\left(F^{*} T N\right) c_{1}(T M)-c_{2}(T M)+c_{1}^{2}(T M)
\end{aligned}
$$

и т. д. Отображение $F$ имеет в данной точке особенность, если его дифференщиал $d F$ вырожден в этой точке. Особенности классифицируются по типам. Замыкание множества точек, в которых $F$ имеет особенность данного типа, определяет, по двойственности Пуанкаре, класс когомологий в $H^{*}(M)$. Из теории Тома следует, что этот класс выражается в виде многочлена от классов Черна отображения $F$, причем коэффициенты многочлена зависят лишь от типа особенности, а не от многообразий $M$ и $N$ или отображения $F$.

Как понятие общего отображения, так и понятие типа особенности требуют уточнения, которое можно найти, например, в [13]. Приведем лишь простейший пример - особенность $A_{1}$ : отображение имеет в данной точке особенность $A_{1}$, если ранг его дифференциала в этой точке меньше максимального на единицу, причем ограничение отображения на ядро дифференциала невырождено. Для общего отображения множество точек, в которых оно имеет особенность $A_{1}$, является подмногообразием комплексной коразмерности 1. Класс когомологий его замыкания совпадает с $c_{1}(F)=c_{1}\left(F^{*} T N\right)-c_{1}(T M)$, что легко увидеть, рассматривая поднятие на $M$ ненулевой дифференциальной формы старшей степени на $N$. Уже в простейших случаях полученное выражение для класса $A_{1}$ приводит к нетривиальным результатам. Предположим, например, что размерность обоих многообразий равна единище, т. е. мы имеем дело с разветвленным накрытием степени, скажем $n$, одной комплексной кривой другой комплексной кривой. Тогда мы получаем формулу Римана-Гурвица для числа точек ветвления общего разветвленного накрытия

$$
c_{1}(F)=n\left(2-2 g_{N}\right)-\left(2-2 g_{M}\right)=2(n-1)-2\left(n g_{N}-g_{M}\right),
$$

где $g_{M}$ и $g_{N}-$ соответственно род накрывающей и род накрываемой кривой. 
Аналогичное утверждение для мультиособенностей состоит в том, что замыкание в $N$ множества точек, в прообразах которых $F$ имеет особенности предписанных типов, выражается в виде универсального многочлена от прямых образов $F_{*}\left(\left(c_{1}(F)\right)^{k_{1}}\left(c_{2}(F)\right)^{k_{2}} \ldots\right) \in H^{*}(N)$ мономов от классов Черна отображения $F$. Мы также распространяем этот подход на случай мультимультиособенностей, т. е. подмногообразий в пространствах модулей функций, состоящих из функций с предписанным набором мультиособенностей.

Методы теории универсальных многочленов мы применяем к универсальному отображению над пространством Гурвища, которое мы сейчас определим. Обозначим через $\overline{\mathcal{U}}_{g, n}$ универсальную кривую над $\overline{\mathcal{H}}_{g, n}$, т. е. комплексное орбиобразие вместе с отображением $\overline{\mathcal{U}}_{g, n} \rightarrow \overline{\mathcal{H}}_{g, n}$, слой которого над точкой $f \in \overline{\mathcal{H}}_{g, n}$ является кривой определения функции $f$ (точнее говоря, фактором этой кривой по модулю действия группы автоморфизмов отображения $f$ ). Еще одно многообразие представляет собой просто прямое произведение $\mathbb{C} P^{1} \times \overline{\mathcal{H}}_{g, n}$, в первом сомножителе которого зафиксирована координата. Универсальное отображение над пространством Гурвица переводит точку универсальной кривой $\overline{\mathcal{U}}_{g, n}$ в значение соответствующей функции в этой точке. Оно расслоено над пространством Гурвица.

ЗАмЕЧАнИЕ 1.5. Как хорошо известно, универсальная кривая над пространством модулей стабильных кривых $\overline{\mathcal{M}}_{g, n}$ совпадает с пространством модулей $\overline{\mathcal{M}}_{g, n+1}$ стабильных кривых с $n+1$ отмеченными точками. Действительно, точку слоя универсальной кривой можно считать $(n+1)$-ой отмеченной точкой вдоль слоя. Для пространств Гурвища имеется похожая конструкция.

Группа $\mathbb{C}^{*}$ ненулевых комплексных чисел по умножению действует на каждом из трех пространств $\overline{\mathcal{H}}_{g, n}, \overline{\mathcal{U}}_{g, n}$ и $\mathbb{C} P^{1} \times \overline{\mathcal{H}}_{g, n}$ умножением функции на константу. На $\overline{\mathcal{U}}_{g, n}$ это действие послойно тривиально, а на слое $\mathbb{C} P^{1}$ прямого произведения группа $\mathbb{C}^{*}$ действует умножением на константу (это действие корректно определено благодаря тому, что мы зафиксировали координату в слое). Универсальное отображение эквивариантно относительно описанных действий, а страты в пространстве Гурвища инвариантны относительно такого действия. Ограничивая и универсальную кривую, и прямое произведение на дополнение к множеству неподвижных точек действия на $\overline{\mathcal{H}}_{g, n}$ и факторизуя полученные пространства по действию группы, мы приходим к треугольнику пространств и отображений, которьй и будет служить основным объектом нашего исследования. Заметим, что поскольку действие группы $\mathbb{C}^{*}$ на слоях прямого произведения $\mathbb{C} P^{1} \times \overline{\mathcal{H}}_{g, n}$ нетривиально, фактор по нему не совпадает с прямым произведением $\mathbb{C} P^{1} \times P^{\frac{g}{\mathcal{H}}} g, n ;$ напротив, он изоморфен проективизации векторного расслоения $\mathbb{C} \oplus \mathcal{O}(1)$ ранга два над $P \overline{\mathcal{H}}_{g, n}$.

Введем упрошенные обозначения: $B_{g, n}$ - для проективизированного пространства Гурвища $P \overline{\mathcal{H}}_{g, n}, X_{g, n}$ - для фактора универсальной кривой (который, в свою очередь, является универсальной кривой над проективизированным пространством Гурвица), $Y_{g, n}$ - для фактора прямого произведения, $p_{g, n}$ (соответственно, $\left.q_{g, n}\right)$ - для проекции пространства $X_{g, n}\left(\right.$ соответственно, $\left.Y_{g, n}\right)$ на $B_{g, n}$ и $f_{g, n}-$ для факторизованного универсального отображения, $f_{g, n}: X_{g, n} \rightarrow Y_{g, n}$. Други- 
ми словами, мы рассматриваем коммутативньй треугольник

$$
\begin{gathered}
X_{g, n} \stackrel{f_{g, n}}{\longrightarrow} \quad Y_{g, n} \\
p_{g, n} \searrow \quad \swarrow q_{g, n} \\
B_{g, n}
\end{gathered}
$$

Сначала мы хотим показать, что относительные классы Черна универсального отображения можно выразить через небольшое число "основных" классов из $H^{*}\left(X_{g, n}\right)$, а затем описать страты в пространстве $B_{g, n}$ в терминах прямых образов многочленов от этих основных классов. Это возможно, поскольку число типов особенностей отображения $f_{g, n}$ невелико, а сами типы не зависят от $g$ и $n$, что и приводит к универсальным формулам.

Отметим, что поскольку пространство Гурвица $B_{g, n}$ является пространством модулей стабильных мероморфных функций степени $n$ на кривых рода $g$, a $X_{g, n}-$ универсальная кривая над $B_{g, n}$, всякое семейство стабильных мероморфных функций степени $n$ на кривых рода $g$ индуцировано из проекции $p_{g, n}: X_{g, n} \rightarrow B_{g, n}$. В частности, линейные соотношения на классы когомологий, справедливые в $X_{g, n}$ и $B_{g, n}$, сохраняются в произвольном семействе.

1.6. Структура статьи. В $\S 2$ мы анализируем локальные особенности универсального отображения и вводим основные классы когомологий. В $§ 3$ мы показываем, что относительные классы Черна универсального отображения действительно выражаются через основные классы. Затем мы выводим выражения для некоторых стратов в $B_{g, n}$ в терминах прямых образов многочленов от основных классов. В $\S 4$ проведены вычисления степеней стратов.

Авторы благодарны Д. Загиру, Д. Звонкину и Б.Лассу за полезные обсуждения. Работа над статьей была завершена во время посешения вторым автором Института математики Макса Планка в Бонне, Германия, в январе-феврале 2004 г.

\section{§2. Особенности расслоенных отображений с одномерными слоями}

Относительные классы Черна универсального отображения $f_{g, n}$ тесно связаны с его особенностями. Поскольку отображение $f_{g, n}$ расслоено над базой $B_{g, n}$, причем слои одномерны, эти особенности оказываются не очень сложными. Классификация простых изолированных особенностей расслоенных отображений с одномерными слоями [5] включает особенности типов $A_{k}$ и $I_{k, l}$. Такие особенности реализуются для отображений $f_{g, n}$. Однако помимо изолированных особенностей (за исключением простейших случаев $g=0, n=2$ или $n=3$ ) эти отображения имеют и неизолированные. Последние возникают в том случае, если в слое, на котором функция определена, есть неприводимая компонента, ограничение функции на которую постоянно. Отметим, что такая функция не обязательно нестабильна: если род неприводимой компоненты не меньше единицы или она рациональна, но на ней есть не менее трех узлов, то постоянство функции на такой компоненте не противоречит ее стабильности. Разумеется, полное изучение пространств Гурвица при нашем подходе требует анализа неизолированных особенностей, которого мы не приводим, а значит, нам удается получить лишь частичные результаты. 
Обсуждению неизолированных особенностей мы планируем посвятить отдельную статью.

2.1. Локальные особенности. Рассмотрим коммутативную треугольную диаграмму пространств и голоморфных отображений вида

$$
\begin{aligned}
\mathbb{C}^{m+1} & \stackrel{f}{\longrightarrow} \mathbb{C} \times \mathbb{C}^{m} \\
p \searrow & \mathbb{C}^{m}
\end{aligned}
$$

где $q$ - проекция прямого произведения на второй сомножитель. Мы предполагаем, что слои отображения $p$ одномерные (хотя и необязательно гладкие). Ограничивая $f$ на слои отображения $p$, мы получаем семейство функций на (некомпактных) кривых. Естественная "правая" эквивалентность таких диаграмм допускает произвольные замены координат в базе $\mathbb{C}^{m}$, а также послойные замены координат в пространстве $\mathbb{C}^{m+1}$, расслоенные над выбранной заменой координат в базе. Мы расширяем эту группу эквивалентностей, позволяя умножать функцию на константу, голоморфно зависящую от точки базы. Такое расширение, не влияя на классификацию особенностей, приводит к более богатой теории характеристических классов.

Отображение $q$ является заданной проекцией, а $p$ представляет собой композицию отображений $f$ и $q$, поэтому особенности диаграммы полностью определяются особенностями отображения $f$. Точка $x \in \mathbb{C}^{m+1}$ является локальной особенностью отображения $f$, если ранг его дифференциала в точке $x$ меньше, чем $m+1$. Сделаем следуюшие два предположения:

- отображение $f$ имеет только общие особенности, т. е. дополнение к множеству таких особенностей в пространстве струй диаграмм является открытым плотным подмножеством;

- проекция $p: \mathbb{C}^{m+1} \rightarrow \mathbb{C}^{m}$ не имеет особенностей, сложнее морсовской складки $(x, y) \mapsto x y$.

Для интересующего нас универсального отображения второе предположение выполняется, а первое не выполняется: ограничение функции на слой может оказаться константой на некоторой неприводимой компоненте слоя. В результате получаемые нами формулы для универсального отображения оказываются верными "с точностью до некоторых классов с носителями на подмногообразии неизолированных особенностей". В случае рациональных функций коразмерность подмногообразия неизолированных особенностей равна трем, что позволяет нам получить точные формулы для стратов вплоть до коразмерности два.

При сделанньх вьше предположениях отображение $f$ может иметь особенности следующих типов:

1) особенности типа $A_{k}, k=1,2, \ldots$, которые реализуются в тех гладких точках слоев отображения $p$, где ограничение отображения $f$ на слой приводится к виду $x \mapsto x^{k+1}$ в подходящей локальной координате $x$ вдоль слоя;

2 ) особенности типа $I_{k, l}, k \geqslant l \geqslant 1$, которые реализуются в тех особых точках слоев отображения $p$, где ограничение функции $f$ на плоскость с локальными 
координатами $x, y$, в которых $p$ имеет вид $(x, y) \mapsto x y$, вьглядит как $f:(x, y) \mapsto$ $\left(x^{k}+y^{l}, x y\right)$.

В таких точках можно выбрать координаты на базе $\mathbb{C}^{m}$, в которых $f$ представляется в виде прямой суммы тождественного отображения и миниверсальных разверток, которые для случаев $A_{k}$ и $I_{k, l}$ выглядят следующим образом:

1) $A_{k}:\left(x, b_{2}, \ldots, b_{k}\right) \mapsto\left(x^{k+1}+b_{2} x^{k-1}+\cdots+b_{k} x, b_{2}, \ldots, b_{k}\right)$;

2) $I_{k, l}:\left(x, y, a_{1}, \ldots, a_{k-1}, b_{1}, \ldots, b_{l-1}\right) \mapsto\left(x^{k}+a_{1} x^{k-1}+\cdots+a_{k-1} x+y^{l}+\right.$ $\left.b_{1} y^{l-1}+\cdots+b_{l-1} y, x y, a_{1}, \ldots, a_{k-1}, b_{1}, \ldots, b_{l-1}\right)$.

Мы интерпретируем эти развертки как глобально заданные полиномиальные отображения комплексных векторных пространств одинаковой размерности. В случае $A_{k}$ (соответственно, $I_{k, l}$ ) такая развертка представляет собой универсальное отображение универсальной кривой над пространством модулей рациональных функций с одним полюсом порядка $k+1$ (соответственно, двух полюсов порядков $k$ и $l$ ) [14]. Точнее говоря, речь идет не о пространстве модулей, а о некотором конечнократном накрытии над этим пространством, кратность которого равна $k+1$ в случае $A_{k}$ и $k l$ в случае $I_{k, l}$. Поэтому в выбранных выше координатах умножение функции на константу задает умножение рациональной функции типа $A_{k}$ (соответственно, $\left.I_{k, l}\right)$ на элемент $\lambda^{k+1} \in \mathbb{C}^{*}\left(\right.$ соответственно, $\lambda^{k l}$ ), что после замены $x \mapsto \lambda x$ определяет на развертках действие группы $\mathbb{C}^{*}$ по формулам:

1) для особенности $A_{k}$

$$
\lambda:\left(x, b_{2}, \ldots, b_{k}\right) \mapsto\left(\lambda x, \lambda^{2} b_{2}, \ldots, \lambda^{k} b_{k}\right), \quad \lambda \in \mathbb{C}^{*}
$$

2) для особенности $I_{k, l}$

$$
\begin{aligned}
& \lambda:\left(x, y, a_{1}, \ldots, a_{k-1}, b_{1}, \ldots, b_{l-1}\right) \\
& \quad \mapsto\left(\lambda^{l} x, \lambda^{k} y, \lambda^{l} a_{1}, \ldots, \lambda^{l(k-1)} a_{k-1}, \lambda^{k} b_{1}, \ldots, \lambda^{k(l-1)} b_{l-1}\right), \quad \lambda \in \mathbb{C}^{*} .
\end{aligned}
$$

2.2. Характеристические классы особенностей. Предположим, что диаграмма (2.1) имеет в начале координат особенность типа $A_{k}$. Без ограничения общности можно предполагать, что $k=m+1$, а $f$-просто выписанная выше миниверсальная развертка особенности $A_{k}$. Пространство $\mathbb{C}^{k}$ из левого верхнего угла диаграммы (2.1) содержит подмногообразия, отвечающие всем особенностям $A_{i}$ при $i \leqslant k$. Эти подмногообразия инвариантны относительно действия группы $\mathbb{C}^{*}$, поэтому особенности $A_{i}$ отвечает подмногообразие $\left[A_{i}\right]$ во взвешенном проективном пространстве $\mathbb{C} P^{(k)}=\mathbb{C} P_{1,2, \ldots, k}$, полученном факторизацией проколотого пространства $\mathbb{C}^{k} \backslash\{0\}$ по описанному выше действию группы $\mathbb{C}^{*}$, а значит, и класс когомологий $\left[A_{i}\right] \in H^{2 i}\left(\mathbb{C} P^{(k)}\right)$. Обозначим через $\Sigma \in H^{2}\left(\mathbb{C} P^{(k)}\right)$ характеристический класс особенности $A_{1}$, т. е. первый класс Черна отображения $f$, а через $\Psi \in H^{2}\left(\mathbb{C} P^{(k)}\right)$ - характеристический класс действия группы $\mathbb{C}^{*}$.

ТЕОРема 2.1. Характеристические классы особенностей $\left[A_{i}\right]$ в развертках особенностей $A_{k}$ выражсаются через $\Sigma$ и по формуле

$$
\left[A_{i}\right]=P_{i}(\Sigma, \Psi)=\Sigma(2 \Sigma-\Psi)(3 \Sigma-2 \Psi) \ldots(i \Sigma-(i-1) \Psi) .
$$

В частности, выражения для этих классов через $\Psi, \Sigma$ не зависят от порядка $k$ деформируемой особенности $A_{k}$. 
ДокАЗАТЕльСтво. Обозначим через $t \in H^{2}\left(\mathbb{C} P^{(k)}\right)$ такой класс когомологий, что $\Psi=(k+1) t$. Тогда $t$ служит образуюшей кольца когомологий $H^{*}\left(\mathbb{C} P^{(k)}\right)$. В силу квазиоднородности $\Sigma=k t$. Выражение для класса $\left[A_{i}\right]$ через $t$ и $k$ должно иметь вид

$$
\left[A_{i}\right]=c_{i} \cdot(k)_{i} t^{i}
$$

для некоторой константы $c_{i}$. Здесь символом Похгаммера $(k)_{i}$ обозначен многочлен от $k$ степени $i$ :

$$
(k)_{i}=k(k-1)(k-2) \ldots(k-i+1) .
$$

Действительно, из теоремы Тома и доказываемого ниже следствия 3.4 вытекает, что класс $\left[A_{i}\right]$ допускает некоторое универсальное представление в виде однородного многочлена от $\Psi$ и $\Sigma$. Подставляя в этот однородный многочлен выражения для $\Psi$ и $\Sigma$ через $t$ и $k$, мы заключаем, что класс $\left[A_{i}\right]$ пропорционален $t^{i}$, причем коэффициент пропорциональности является многочленом от $k$ степени $i$, который обрашается в нуль при всех $k<i$. Для случая $i=k$ мы имеем $\left[A_{i}\right]=i ! t^{i}$, что дает $c_{i}=1$. Теперь выражение для $A[i]$ через $\Psi$ и $\Sigma$ получается в результате подстановки $t=\Psi-\Sigma, k=\Sigma /(\Psi-\Sigma)$. Теорема доказана.

Миниверсальная развертка особенности $I_{k, l}$ содержит как страты, отвечающие особенностям $A_{i}$ при $i<k+l$, так и страты, отвечаюшие особенностям типов $I_{i, j}$ при $i \leqslant k$ и $j \leqslant l$. Эти страты также инвариантны относительно действия группы $\mathbb{C}^{*}$. Факторизуя по этому действию, мы получаем взвешенное проективное пространство $\mathbb{C} P^{(k, l)}=\mathbb{C} P_{l, l, 2 l, \ldots,(k-1) l, k, k, 2 k, \ldots,(l-1) k}$, и все классы когомологий, которые мы рассматриваем ниже, лежат в когомологиях этого пространства.

Особенности типов $I_{i, j}$ лежат в подмногообразии $\Delta$ особых точек слоев проекции $p$. В выбранных выше координатах это подмногообразие задается уравнением $x=y=0$. Добавим к классам $\Psi, \Sigma \in H^{2}\left(\mathbb{C} P^{(k, l)}\right)$ класс $\Delta \in H^{4}\left(\mathbb{C} P^{(k, l)}\right)$. Рассмотрим также нормальное расслоение к $\Delta$ и обозначим через $N$ его первьй класс Черна. Класс $\Delta$ можно интерпретировать как второй класс Черна этого нормального расслоения, причем с классами $N$ и $\Delta$ можно обращаться как с (коммутирующими) операторами, действующими на $\Delta$. Еще менее формально можно считать класс $N$ элементом вторых когомологий, $N \in H^{2}\left(\mathbb{C} P^{(k, l)}\right)$ (смысл которому придается только при его пересечении с $\Delta$ ). Нас интересуют выражения для особенностей типов $A_{i}$ в развертках особенностей типов $I_{k, l}$ в терминах классов $\Sigma, \Psi, \Delta, N$.

Tеорема 2.2. Существуют такие выражсения для классов $\left[A_{i}\right]$ в развертках особенностей $I_{k, l}$ в виде квазиоднородных многочленов степени $i$ от переменных $\Sigma, \Psi, \Delta, N$, что:

i) эти многочлень универсальны, т.е. они зависят от $i$, но не от $k$ и $l$;

ii) многочлен, соответствующий классу $\left[A_{i}\right]$, представляется в виде суммы двух многочленов, первый из которых совпадает с определенным выше многочленом $P_{i}(\Sigma, \Psi)$, а носитель второго сосредоточен на страте $\Delta$ двойных точек;

iii) всякий многочлен с носителем на $\Delta$ можно представить в виде многочлена от $\Delta, N u \Psi$. 
Первые два утверждения вытекают из теоремы Тома и следствия 3.4 , а последнее является следствием очевидного соотношения $\Sigma \Delta=\Psi \Delta$.

Обозначим многочлен, отвечаюший классу $\left[A_{i}\right]$, через $R_{i}$. Он имеет вид $R_{i}=$ $P_{i}+Q_{i-2} \Delta$, где многочлен $P_{i}$ уже известен, а $Q_{i-2}=Q_{i-2}(\Delta, N, \Psi)$ представляет собой взвешенно однородньй многочлен степени $i-2$. Для этих многочленов нам не удалось найти явной формулы, однако их можно вычислить, воспользовавшись методом неопределенных коэффициентов. Применим этот метод при малых $i$.

Аналогично рассмотренной выше ситуации, всякий элемент из $H^{*}\left(\mathbb{C} P^{(k, l)}\right)$ представляется в виде многочлена от $t=\frac{\Psi}{k l}$. Зная веса квазиоднородности, мы заключаем, в частности, что

$$
\Psi=\Sigma=k l t, \quad N=(k+l) t, \quad \Delta=k l t^{2} .
$$

Найдем, воспользовавшись этими представлениями, коэффициенты многочленов $Q_{0}$ и $Q_{1}$. Мы знаем, что $Q_{0}=a$ для некоторой постоянной $a$ и что страт $A_{2}$ в развертке особенности $I_{1,1}$ пуст. Для случая $k=l=1$ имеем $\Psi=\Sigma=t, \Delta=t^{2}$, откуда

$$
R_{2}=\Sigma(2 \Sigma-\Psi)+a \Delta=t^{2}+a t^{2}=0 .
$$

Поэтому $a=-1$, т.е.

$$
R_{2}=\Sigma(2 \Sigma-\Psi)-\Delta
$$

Аналогично, $Q_{1}=a N+b \Psi$ для некоторых постоянных $a$ и $b$. Воспользовавшись тем, что страт $A_{3}$ пуст в развертках каждой из особенностей $I_{1,1}$ и $I_{2,1}$, мы получаем следующую систему линейных уравнений:

$$
\begin{aligned}
1+2 a+b & =0 \\
8+6 a+4 b & =0
\end{aligned}
$$

(воспользовавшись однородностью, мы поделили оба уравнения на $t^{3}$ ), откуда $a=2, b=-5$, т. е.

$$
Q_{1}=2 N-5 \Psi
$$

Используя аналогичные вычисления, получаем

$$
\begin{aligned}
Q_{2}= & -\left(6 N^{2}-15 N \Psi+15 \Psi^{2}-8 \Delta\right), \\
Q_{3}= & 24 N^{3}-62 N^{2} \Psi+63 N \Psi^{2}-35 \Psi^{3}-60 N \Delta+84 \Psi \Delta, \\
Q_{4}= & -\left(120 N^{4}-322 N^{3} \Psi+343 N^{2} \Psi^{2}-196 N \Psi^{3}+70 \Psi^{4}\right. \\
& \left.-432 N^{2} \Delta+812 N \Psi \Delta-469 \Psi^{2} \Delta+180 \Delta^{2}\right) .
\end{aligned}
$$

Отметим, что число неопределенных коэффициентов в разложении особенности $A_{i}$ равно $\left[i^{2} / 4\right]$, где квадратные скобки обозначают целую часть числа, что в точности совпадает с числом особенностей типа $I_{k, l}$, миниверсальные развертки которых не содержат страта $A_{i}$ по соображениям размерности. 
2.3. Остаточные многочлены для мультиособенностей. Зафиксируем разбиение $\alpha=1^{m_{1}} 2^{m_{2}} \ldots$ (в котором лиш конечное число показателей отлично от нуля). Рассмотрим множество точек $y$ в пространстве $\mathbb{C} \times \mathbb{C}^{m}$ в правом верхнем углу коммутативного треугольника (2.1), обладающих следующими свойствами:

- слой $p^{-1}(q(y))$ проекции $p$ неособ;

- ограничение отображения $f$ на этот слой имеет среди прообразов точки $y$ в точности $m_{1}$ экземпляров особенности $A_{1}$, в точности $m_{2}$ экземпляров особенности $A_{2}$ и так далее (помимо особенностей предписанных типов точка $y$ может иметь и неособые прообразы, количество которых определяется степенью отображения $f$ ).

Обозначим класс замыкания этого множества, умноженный на порядок $m_{1} ! m_{2} ! \ldots$ группы автоморфизмов разбиения $\alpha$, через $A_{\alpha}(f) \subset \mathbb{C} \times \mathbb{C}^{m}(f)=$ $A_{1} m_{1} 2^{m_{2}} \ldots(f)$. (У Множение на порядок группы автоморфизмов позволяет избавиться от дробей в последуюших явных вычислениях классов.)

Если бы прямые образы $f_{*} R_{k}$ подмногообразий $R_{k}=A_{k}$ пересекались трансверсально, то классы $A_{1} m_{1} 2^{m_{2}} \ldots(f)$ описьвались бы коэффициентами при $\frac{t_{1}^{m_{1}}}{m_{1} !} \frac{t_{2}^{m_{2}}}{m_{2} !} \ldots$ в экспоненте производящей функции $f_{*}\left(R_{1} t_{1}+R_{2} t_{2}+\ldots\right)$. (Появление факториалов $m_{i}$ ! в знаменателях отражает возможность перенумеровывать прообразы с одинаковыми типами особенностей.) Нетрансверсальность пересечения приводит к необходимости введения поправок - остаточных многочленов [10].

Рассмотрим экспоненциальную производящую функцию

$$
\mathcal{A}(f)=\sum\left[A_{1} m_{1} 2^{m_{2}} \ldots(f)\right] \frac{t_{1}^{m_{1}}}{m_{1} !} \frac{t_{2}^{m_{2}}}{m_{2} !} \ldots,
$$

где суммирование ведется по всем разбиениям $\alpha=1^{m_{1}} 2^{m_{2}} \ldots$, включая пустое разбиение, а квадратными скобками обозначены классы когомологий, двойственные по Пуанкаре соответствующим многообразиям. Введем также экспоненциальную производящую функцию от бесконечного набора переменных $t_{1}, t_{2}, \ldots$ с формальными коэффициентами $R_{\alpha}$, где $\alpha$ принимает значения из множества всех ненулевых разбиений:

$$
\mathcal{R}\left(t_{1}, t_{2}, \ldots\right)=\sum R_{1} m_{1} m_{2} \frac{t_{1}^{m_{1}}}{m_{1} !} \frac{t_{2}^{m_{2}}}{m_{2} !} \ldots
$$

ПРЕДЛОЖЕНИЕ 2.3. Существуют взвешенно однороднье многочлены $R_{\alpha}$ от классов $\Sigma, \Psi, \Delta, N$ такие, что после их подстановки в качестве коэффичиентов в производящие ряды $\mathcal{R}$ классы когомологий стратов $A_{1} m_{1} 2^{m_{2}} \ldots(f)$ совпадают с коэффициентами при мономах $\frac{t_{1}^{m_{1}}}{m_{1} !} \frac{t_{2}^{m_{2}}}{m_{2} !} \ldots$ в әкспоненте $\exp \left(f_{*} \mathcal{R}\right)$ :

$$
\mathcal{A}(f)=\exp \left(f_{*} \mathcal{R}\left(t_{1}, t_{2}, \ldots\right)\right) .
$$

Здесь символом $f_{*}$ обозначен гомоморфизм Гизина, т. е. гомоморфизм прямого образа в когомологиях факторпространств по действию групшы $\mathbb{C}^{*}$. Это отображение является гомоморфизмом лишь аддитивной, не мультипликативной структуры. Введенные таким образом многочлены $R_{\alpha}$ называются остаточныцми многочленами для мультиособенностей. Разумеется, нет ничего удивительного в том, что многочлены $R_{k^{1}}$ совпадают с многочленами $R_{k}$, введенныги в п. 2.2 (действительно, у соответствуюших точек есть лишь один особый прообраз). 
ДоКАЗАТЕЛЬСТво. Наиболее существенной частью предложения является утверждение о том, что поправка $f_{*}\left(R_{1} m_{1} 2^{m_{2}} \ldots\right)$ в выражении для класса $\left[A_{\alpha}\right]$ линейна по прямым образам гомоморфизма Гизина. Основное наблюдение, позволяющее доказать это утверждение, состоит в том, что универсальное выражение для классов мультиособенностей справедливо и в том случае, когда кривые области определения мероморфных функций несвязны.

Применим это наблюдение к семейству отображений кривых общего вида

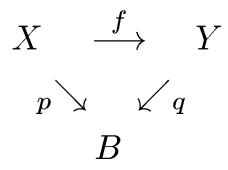

Рассмотрим отображение $f^{(d)}: X^{(d)} \rightarrow Y$, где через $X^{(d)}$ обозначено расслоенное над базой $B$ пространство, представляющее собой несвязное объединение $d$ копий пространства $X, X^{(d)}=X_{1} \sqcup \cdots \sqcup X_{d}, X_{i}=X$, а $f^{(d)}$ - отображение, ограничение которого на $i$-ю копию $X_{i}$ совпадает с $f$. Точнее говоря, мы берем в качестве ограничения отображения $f^{(d)}$ на $X_{i}$ несколько возмущенную копию отображения $f$, что необходимо для приведения отображения $f^{(d)}$ в общее положение.

Поскольку выражение для классов мультиособенностей универсально, его можно применять и к каждому из построенных отображений $f^{(d)}$. Нетрудно видеть, что

$$
\mathcal{A}\left(f^{(d)}\right)=(\mathcal{A}(f))^{d} .
$$

Действительно, всякое разбиение $\alpha$, соответствующее мультиособенности отображения $f^{(d)}$, расщепляется на $d$ подразбиений, каждое из которых соответствует мультиособенности одной из компонент отображения $f^{(d)}$. Комбинаторные коэффициенты этих разбиений как раз и соответствуют возведению в степень $d$. Мы заключаем, что

$$
\log \mathcal{A}\left(f^{(d)}\right)=d \log \mathcal{A}(f) .
$$

С другой стороны, прямой образ всякого монома от основных классов относительно отображения $f^{(d)}$ равен, очевидно, $d$-кратному прямому образу этого монома относительно $f$. Следовательно, всякий коэффициент в производящей функции $\log \mathcal{A}(f)$ не содержит никаких произведений образов основных классов (такие произведения дали бы вклад, степень которого по $d$ больше 1), а значит, линеен. Предложение доказано.

Для вычисления остаточных многочленов мы должны знать действие гомоморфизма $f_{*}$ на интересующих нас когомологических классах. Образом отображения $f$ также является взвешенное проективное пространство, и его кольцо когомологий порождено классом гиперплоскости, который мы также обозначаем через $t$. Поэтому $f_{*}$ имеет вид $f_{*}: t^{i} \mapsto c t^{i}$ для некоторой постоянной $c$. Эта постоянная не может быть ни чем иным, как постоянной $f_{*} 1$, т. е. степенью отображения $f$. Если отображение $f$ - миниверсальная развертка особенности типа $A_{k}$, то его степень равна $k+1$.

Начнем с вычисления многочленов $R_{\alpha}$ для стандартных разверток особенностей типа $A_{k}$ (т. е. в присутствии лишь особенностей типа $A$ ). Эти многочлены зависят лишь от классов $\Sigma$ и $\Psi$. Их можно вычислить следуюшим образом. 
После подстановки $\Sigma=k t, \Psi=(k+1) t$ в производящую функцию $\mathcal{R}$ мы должны получить

$$
\begin{gathered}
\exp \left((k+1) \mathcal{R}\left(t_{1}, t_{2}, \ldots\right)\right)=1+(k+1)_{2} \frac{t_{1}}{1 !} t+\left((k+1)_{3} \frac{t_{2}}{1 !}+(k+1)_{4} \frac{t_{1}^{2}}{2 !}\right) t^{2} \\
+\left((k+1)_{4} \frac{t_{3}}{1 !}+(k+1)_{5} \frac{t_{1}}{1 !} \frac{t_{2}}{1 !}+(k+1)_{6} \frac{t_{1}^{3}}{3 !}\right) t^{3}+\ldots
\end{gathered}
$$

Действительно, коэффициент при мономе $\frac{t_{1}^{i_{1}}}{i_{1} !} \frac{t_{2}^{i_{2}}}{i_{2} !} \ldots$ в экспоненте должен иметь вид $t^{i_{1}+2 i_{2}+3 i_{3}+\ldots}$ с коэффициентом, который представляет собой многочлен от $k$, обрашаюшийся в нуль при всех $k$ вплоть до коразмерности соответствуюшего страта, $k=-1,0,1, \ldots, 2 i_{1}+3 i_{2}+\cdots-2$.

Отметим, что правую часть равенства $(2.3)$ удобно переписать в виде ${ }^{1}$

$$
\exp \left((k+1) \mathcal{R}\left(t_{1}, t_{2}, \ldots\right)\right)=\left.e^{\tau_{1} \tau+\tau_{2} \tau^{2}+\tau_{3} \tau^{3}+\cdots} s^{k+1}\right|_{s=1} .
$$

Здесь $s$ - дополнительная переменная, коммутируюшая со всеми $t_{i}$ и $t$, и мы используем обозначения

$$
\tau=t \frac{d}{d s}, \quad \tau_{i}=t_{i} \frac{d}{d s} .
$$

Теперь остаточные многочлены $R_{\alpha}$ несложно подсчитать, взяв логарифм правой части равенства $(2.3)$, поделив его на $(k+1)$ и выполнив подстановку $t=\Psi-\Sigma$, $k=\Sigma /(\Psi-\Sigma)$. Для малых коразмерностей получаем

$$
\begin{aligned}
R_{1^{2}} & =-2 \Sigma(3 \Sigma-\Psi), \\
R_{1^{1} 2^{1}} & =-6 \Sigma(2 \Sigma-\Psi)^{2}, \\
R_{1^{3}} & =8 \Sigma\left(15 \Sigma^{2}-13 \Sigma \Psi+3 \Psi^{2}\right), \\
R_{1^{1} 3^{1}} & =-4 \Sigma(5 \Sigma-3 \Psi)(3 \Sigma-2 \Psi)(2 \Sigma-\Psi), \\
R_{2^{2}} & =-3 \Sigma(2 \Sigma-\Psi)\left(20 \Sigma^{2}-25 \Sigma \Psi+8 \Psi^{2}\right), \\
R_{1^{2} 2^{1}} & =24 \Sigma(2 \Sigma-\Psi)\left(15 \Sigma^{2}-17 \Sigma \Psi+5 \Psi^{2}\right), \\
R_{1^{4}} & =-48 \Sigma\left(105 \Sigma^{3}-160 \Sigma^{2} \Psi+84 \Sigma \Psi^{2}-15 \Psi^{3}\right) .
\end{aligned}
$$

$\Delta$-часть остаточных многочленов в развертках особенностей $I_{k, l}$ можно вычислить рекуррентно, воспользовавшись, как и вьше, методом неопределенных коэффициентов.

Вычислим, например, многочлен $R_{12}$. В силу взвешенной однородности он имеет вид

$$
R_{1^{2}}=2 \Sigma \Psi-6 \Sigma^{2}+a \Delta
$$

для некоторой постоянной $a$ (свободная от $\Delta$ часть нам уже известна), а страт $A_{12}$ представляется как неизвестный коэффициент при $t_{1}^{2} / 2$ ! в $\exp \left(f_{*} \mathcal{R}\right)$ :

$$
\left[A_{1^{2}}\right]=\left(f_{*} R_{1^{1}}\right)^{2}+f_{*} R_{1^{2}}=\left(f_{*} \Sigma\right)^{2}+f_{*}\left(2 \Sigma \Psi-6 \Sigma^{2}+a \Delta\right) .
$$

\footnotetext{
${ }^{1}$ Мы обязаны этим замечанием Д. Загиру.
} 
Степень развертки особенности $I_{k, l}$ равна $k+l$. Подставляя выражения для классов $\Psi, \Delta, \Sigma$ через класс $t$ в развертку особенности $I_{1,1}$ и пользуясь тем, что класс $A_{1^{2}}$ в этой развертке пуст, мы получаем линейное уравнение

$$
2^{2}+4-12+2 a=0
$$

откуда $a=2$, т.е.

$$
R_{1^{2}}=2\left(\Sigma \Psi-3 \Sigma^{2}+\Delta\right)
$$

Аналогичные вычисления для остаточных многочленов в более высоких коразмерностях приводят к следуюшим ответам:

$$
\begin{aligned}
R_{1^{1} 2^{1}}= & -6 \Sigma(2 \Sigma-\Psi)^{2}+6 \Delta\left(3 \Psi-N_{1}\right), \\
R_{1^{3}}= & 8 \Sigma\left(15 \Sigma^{2}-13 \Sigma \Psi+3 \Psi^{2}\right)-8 \Delta(10 \Psi-3 N), \\
R_{1^{1} 3^{1}}= & -4 \Sigma(5 \Sigma-3 \Psi)(3 \Sigma-2 \Psi)(2 \Sigma-\Psi) \\
& +4 \Delta\left(20 \Psi^{2}-17 N \Psi+6 N^{2}-8 \Delta\right), \\
R_{2^{2}}= & -3 \Sigma(2 \Sigma-\Psi)\left(20 \Sigma^{2}-25 \Sigma \Psi+8 \Psi^{2}\right) \\
& +3 \Delta\left(25 \Psi^{2}-21 N \Psi+8 N^{2}-12 \Delta\right), \\
R_{1^{2} 2^{1}}= & 24 \Sigma(2 \Sigma-\Psi)\left(15 \Sigma^{2}-17 \Sigma \Psi+5 \Psi^{2}\right) \\
& -24 \Delta\left(20 \Psi^{2}-15 N \Psi+5 N^{2}-7 \Delta\right), \\
R_{1^{4}}= & -48 \Sigma\left(105 \Sigma^{3}-160 \Sigma^{2} \Psi+84 \Sigma \Psi^{2}-15 \Psi^{3}\right) \\
& +48 \Delta\left(70 \Psi^{2}-48 N \Psi+15 N^{2}-21 \Delta\right) .
\end{aligned}
$$

2.4. Остаточные многочлены для мультимультиособенностей. Теперь нас интересуют многообразия в нижнем углу треугольника (2.1), состоящие из тех точек $b$, для которых ограничение отображения $f$ на слой $p^{-1}(b)$ имеет несколько вырожденных мультиособенностей с предписанными приведенными разбиениями $\alpha_{1}^{m_{1}}, \ldots, \alpha_{c}^{m_{c}}$ (а также необходимое дополнительное число невырожденных особенностей). Показатели здесь указывают кратности соответствуюших разбиений. Обозначим через $A_{\alpha_{1}^{m_{1}}, \ldots, \alpha_{c}^{m_{c}}}(f) \subset \mathbb{C}^{m}$ замыкание множества таких точек, умноженное, аналогично случаю мультиособенностей, на произведение порядков групп автоморфизмов разбиений $\alpha_{i}$ и на порядок группы автоморфизмов набора разбиений $\alpha_{i}$.

Наши вычисления показывают, что справедливо следующее утверждение, которому мы не нашли еше, однако, формального доказательства.

ГИПотеЗА 2.4. При выполнении условий, сформулированных в начале §2, всякий класс мультимультиособенностей задается универсальной полиномиальной комбинацией образов основных классов под действием гомоморфизма Гизина $p_{*}$.

Как и для случая мультиособенностей, только лишь из существования универсальных многочленов вытекает, что они должны иметь довольно специальный вид и выражаться через остаточные многочлены от основных классов. 
Рассмотрим бесконечный набор переменных $s_{1^{2}}, s_{2^{1}}, s_{1^{2}, 1^{2}}, s_{1^{2}, 2^{1}}, s_{2^{1}, 2^{1}}, \ldots$, индексированный наборами разбиений $\alpha$ (упорядоченных, скажем, лексикографически). Определим на множестве этих переменных умножение, положив произведение двух переменных равным сумме таких же переменных, индексы которых представляют собой все наборы разбиений, получаюшиеся объединением некоторых попарно различных разбиений из индекса второй переменной с некоторыми попарно различными разбиениями из индекса первой переменной. Так, например, мы полагаем

$$
s_{\alpha, \beta} s_{\gamma, \delta}=s_{\alpha, \beta, \gamma, \delta}+s_{\alpha \gamma, \beta, \delta}+s_{\alpha, \beta \gamma, \delta}+s_{\alpha \delta, \beta, \gamma}+s_{\alpha, \beta \delta, \gamma}+s_{\alpha \gamma, \beta \delta}+s_{\alpha \delta, \beta \gamma} .
$$

В частности, всякий многочлен от переменных $s_{\alpha_{1}, \alpha_{2}, \ldots}$ равен некоторому линейному многочлену.

Введем производящую функиию для мультимультиособенностей формулой

$$
\mathfrak{A}\left(s_{1^{2}}, s_{2^{1}}, \ldots\right)=\sum \frac{\left[A_{\left.\alpha_{1}^{m_{1}}, \ldots, \alpha_{c}^{m_{c}}\right]}\right]}{\prod \operatorname{Aut}\left(\alpha_{i}\right)} \frac{s_{\alpha_{1}^{m_{1}}, \ldots, \alpha_{c}^{m_{c}}}}{m_{1} ! \ldots m_{c} !} .
$$

Рассмотрим также экспоненциальную производящую функцию с формальньми

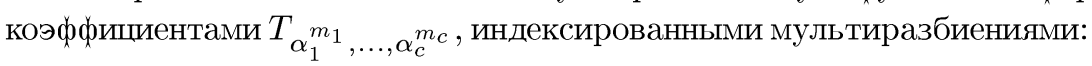

$$
\mathfrak{R}\left(s_{1^{2}}, s_{2^{1}}, \ldots\right)=\sum \frac{T_{\alpha_{1}^{m_{1}}, \ldots, \alpha_{c}^{m_{c}}}}{\prod \operatorname{Aut}\left(\alpha_{i}\right)} \frac{s_{\alpha_{1}^{m_{1}}, \ldots, \alpha_{c}^{m_{c}}}}{m_{1} ! \ldots m_{c} !} .
$$

ПРЕДЛОЖЕНИЕ 2.5. При условии справедливости гипотезы 2.4 если в качестве $T_{\alpha}$ взять многочлены, представляющие классы $A_{\alpha}$, то существуют взвешенно однородные многочлены $R_{\alpha_{1}^{m_{1}}, \ldots, \alpha_{c}^{m_{c}}}$ при $c \geqslant 2$ от классов $\Sigma, \Psi, \Delta, N$ такие, что после подстановки их прямых образов $f_{*} R_{\alpha_{1}, \ldots, \alpha_{c}}$

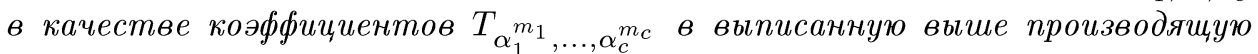
функиию $\Re$ классы когомологий стратов $A_{\alpha_{1}^{m_{1}}, \ldots, \alpha_{c}^{m_{c}}}$ совпадают с коэффициентами при $\frac{s_{\alpha_{1}^{m_{1}}, \ldots, \alpha_{c}^{m_{c}}}}{m_{1} ! \ldots m_{c} !}$ в $\exp \left(q_{*} \mathfrak{R}\right)$, где әкспонента берется относительно описанного выше умножсения переменных $s$.

Это предложение доказывается точно так же, как и предложение 2.3, путем рассмотрения отображения $f^{(d)}$.

Как и выше, многочлены $R_{\alpha_{1}, \ldots, \alpha_{c}}$ можно найти индуктивно с помошью метода неопределенных коэффищиентов. В вычислениях используется то, что ограничение проекции $p$ на $\Sigma$ - собственное отображение, а носители всех интересующих нас когомологических классов сосредоточены на $\Sigma$ и, следовательно, гомоморфизм $p_{*}$, выраженный через базисный класс $t$, сводится к умножению на степень ограничения $\left.p\right|_{\Sigma}$. Последняя степень совпадает с числом критических точек функции $f$ на обшем слое и равна $k+1$ для особенности $A_{k}$ и $k+l$ для особенности $I_{k, l}$. Вот первые нетривиальные результаты вычислений:

$$
\begin{aligned}
& R_{2^{1}, 2^{1}}=-2 \Sigma(2 \Sigma-\Psi)(5 \Sigma-3 \Psi)+\Delta(14 \Psi-5 N) \\
& R_{2^{1}, 1^{2}}=6 \Sigma(2 \Sigma-\Psi)(5 \Sigma-2 \Psi)-6 \Delta(7 \Psi-2 N) \\
& R_{1^{2}, 1^{2}}=-6 \Sigma\left(30 \Sigma^{2}-21 \Psi \Sigma+4 \Psi^{2}\right)+2 \Delta(57 \Psi-14 N) .
\end{aligned}
$$




\section{§3. Относительные классы Черна универсального отображения}

В этом параграфе мы показываем, что относительные классы Черна универсального расслоения действительно принадлежат подкольцу в $H^{*}\left(X_{g, n}\right)$, порожденному основными классами. Отсюда вытекает, что классы $\left[A_{k}\right]$ и остаточные многочлены для мультиособенностей и мультимультиособенностей также принадлежат этому кольцу. Основным инструментом в вычислении относительных классов Черна отображения $f_{g, n}$ служит теорема Гротендика-Римана-Роха. Из нее же вытекают и нетривиальные соотношения на прямые образы Гизина многочленов от основных классов. Напомним сначала эту теорему в той конкретной ситуации, в которой мы будем ее использовать. Наше изложение следует работам $[3],[18]$.

3.1. Теорема Гротендика-Римана-Роха. Теорема Гротендика-РиманаРоха (ГРР) сравнивает характер Черна когерентного пучка над многообразием $M$ с характером Черна образа этого пучка при отображении $P: M \rightarrow A$. Предположим, что оба многообразия $M$ и $A$ компактные и гладкие.

В одной из своих форм (по-видимому, наиболее полезной) формула ГРР вьглядит следуюшим образом:

$$
\operatorname{ch}\left(P_{!} \beta\right)=P_{*}\left(\operatorname{ch}(\beta) \operatorname{td}(P)^{-1}\right) .
$$

Здесь $\beta$ - некоторый когерентный пучок на $M$, ch обозначает характер Черна, a td - класс Тодда морфизма $P$.

Дадим определения понятиям, участвующим в формуле ГРР.

3.1.1. Характер Черна. Характер Черна пучка сечений векторного расслоения, или просто характер Черна векторного расслоения, определяется как сумма экспонент его корней Черна, т. е. формальных корней его многочлена Черна,

$$
\operatorname{ch}(E)=\exp \left(e_{1}\right)+\cdots+\exp \left(e_{r}\right),
$$

где через $r$ обозначен ранг векторного расслоения $E$. В частности, для линейного расслоения $E$ имеем $\operatorname{ch}(E)=\exp \left(c_{1}(E)\right)$.

Как симметрическая функция от корней Черна характер Черна расслоения выражается через его характеристические классы:

$$
\begin{aligned}
\operatorname{ch}(E)= & r+c_{1}+\frac{1}{2}\left(c_{1}^{2}-2 c_{2}\right)+\frac{1}{6}\left(c_{1}^{3}-3 c_{1} c_{2}+3 c_{3}\right) \\
& +\frac{1}{24}\left(c_{1}^{4}-4 c_{1}^{2} c_{2}+4 c_{1} c_{3}+2 c_{2}^{2}-4 c_{4}\right) \\
& +\frac{1}{120}\left(c_{1}^{5}-5 c_{1}^{3} c_{2}+5 c_{1} c_{2}^{2}+5 c_{1}^{2} c_{3}-5 c_{2} c_{3}-5 c_{1} c_{4}+5 c_{5}\right)+\ldots,
\end{aligned}
$$

где через $c_{i}$ обозначена $i$-я элементарная симметрическая функция от корней Черна.

В силу свойств корней Черна для точной последовательности векторных расслоений

$$
0 \longrightarrow E^{\prime} \longrightarrow E \longrightarrow E^{\prime \prime} \longrightarrow 0
$$


справедлива формула Уитни

$$
\operatorname{ch}(E)=\operatorname{ch}\left(E^{\prime}\right)+\operatorname{ch}\left(E^{\prime \prime}\right) .
$$

Формула Уитни позволяет распространить понятие характера Черна на произвольные когерентные пучки. Когерентный пучок на $M$ - это либо пучок сечений некоторого векторного расслоения, либо, более общим образом, пучок, который можно вставить последним нетривиальным членом в точную последовательность пучков, все элементы которой, кроме последнего, - пучки сечений векторных расслоений (такая точная последовательность называется резольвентой для данного пучка). Характер Черна когерентного пучка определяется как альтернированная сумма характеров Черна остальных членов точной последовательности.

Резольвента для структурного пучка подмногообразия $\Sigma \subset M$ коразмерности один в $M$, заданного вложением $i: \Sigma \hookrightarrow M$, выглядит следуюшим образом:

$$
0 \longrightarrow N_{\Sigma}^{\vee} \longrightarrow \mathcal{O}_{M} \longrightarrow i_{*}\left(\mathcal{O}_{\Sigma}\right) \longrightarrow 0
$$

где $N_{\Sigma}$ - линейное расслоение над $M$ такое, что $\Sigma$ совпадает с множеством нулей его сечения. В трубчатой окрестности подмногообразия $\Sigma$ расслоение $N_{\Sigma}$ совпадает с естественным продолжением нормального расслоения к $\Sigma$ в $M$. Из формулы Уитни вытекает равенство

$$
\begin{aligned}
\operatorname{ch}\left(i_{*}\left(\mathcal{O}_{\Sigma}\right)\right) & =\operatorname{ch}\left(\mathcal{O}_{M}\right)-\operatorname{ch}\left(N_{\Sigma}^{\vee}\right)=1-\exp (-\Sigma) \\
& =\frac{(1-\exp (-\Sigma))}{\Sigma} \Sigma=\left(\frac{\Sigma}{1-\exp (-\Sigma)}\right)^{-1} \Sigma
\end{aligned}
$$

причем оператор пересечения с первым классом Черна нормального расслоения можно заменить на $\Sigma$, поскольку согласно предположению подмногообразие $\Sigma$ совпадает с множеством нулей голоморфного сечения нормального расслоения.

Для подмногообразия $Z$ коразмерности два резольвента оказывается более сложной, а именно она имеет вид

$$
0 \longrightarrow \Lambda^{2} N_{Z}^{\vee} \longrightarrow N_{Z}^{\vee} \longrightarrow \mathcal{O}_{M} \longrightarrow i_{*}\left(\mathcal{O}_{Z}\right) \longrightarrow 0
$$

где $\Lambda^{2}$ обозначает кососимметрический квадрат векторного расслоения. Эта последовательность, называемая резольвентой Кошуля, выписана в предположении, что $Z$ является множеством нулей глобально определенного сечения некоторого векторного расслоения $N_{Z}$ над $M$. Мы увидим, что окончательное выражение для $\operatorname{ch}\left(i_{*}\left(\mathcal{O}_{Z}\right)\right)$ определяется ограничением расслоения $N_{Z}$ на $Z$, которое совпадает с нормальным расслоением к $Z$. Более того, можно показать (см. [3]), что полученное выражение справедливо и в отсутствие предположения о том, что нормальное расслоение к $Z$ продолжается на все $M$. Из формулы Уитни имеем

$$
\operatorname{ch}\left(i_{*} \mathcal{O}_{Z}\right)=\operatorname{ch}\left(\Lambda^{2} N_{Z}^{\vee}\right)+\operatorname{ch}\left(\mathcal{O}_{M}\right)-\operatorname{ch}\left(N_{Z}^{\vee}\right)
$$


Ранг виртуального векторного расслоения $N_{Z}^{\vee}$ равен 2 , а его полньй класс Черна равен $c\left(N_{Z}^{\vee}\right)=1-N_{1}+N_{2}$, где $N_{i}=c_{i}\left(N_{Z}\right), i=1,2$. Поэтому его характер Черна имеет вид

$$
\begin{aligned}
\operatorname{ch}\left(N_{Z}^{\vee}\right)=2 & -N_{1}+\frac{1}{2}\left(N_{1}^{2}-2 N_{2}\right)+\frac{1}{6}\left(3 N_{1} N_{2}-N_{1}^{3}\right)+\frac{1}{24}\left(N_{1}^{4}-4 N_{1}^{2} N_{2}+2 N_{2}^{2}\right) \\
& -\frac{1}{120}\left(N_{1}^{5}-5 N_{1}^{3} N_{2}+5 N_{1} N_{2}^{2}\right)+\ldots
\end{aligned}
$$

Векторное расслоение $\Lambda^{2} N_{Z}^{\vee}$ линейно и

$$
c\left(\Lambda^{2} N_{Z}^{\vee}\right)=1-N_{1},
$$

откуда имеем

$$
\operatorname{ch}\left(\Lambda^{2} N_{Z}^{\vee}\right)=\exp \left(-N_{1}\right) .
$$

Сводя эти результаты воедино, мы получаем

$$
\begin{aligned}
\operatorname{ch}\left(i_{*} \mathcal{O}_{Z}\right) & =N_{2}-\frac{1}{2} N_{1} N_{2}+\ldots \\
& =\left(1-\frac{1}{2} N_{1}+\frac{1}{12}\left(N_{1}^{2}-N_{2}\right)-\frac{1}{24}\left(N_{1}^{3}-N_{1} N_{2}\right)+\ldots\right) Z,
\end{aligned}
$$

поскольку и $Z$, и $N_{2}$ отождествляются с классом нулевого сечения нормального расслоения $N_{Z}$.

3.1.2. Класс Тодда. Начнем с определения класса Тодда векторного расслоения, а затем определим класс Тодда отображения. Класс Тодда векторного расслоения $E$ над $M$ - это еще одна симметрическая функция его корней Черна,

$$
\begin{aligned}
\operatorname{td}(E)= & \frac{e_{1}}{1-\exp \left(-e_{1}\right)} \cdots \frac{e_{r}}{1-\exp \left(-e_{r}\right)}=1+\frac{1}{2} c_{1}+\frac{1}{12}\left(c_{1}^{2}+c_{2}\right) \\
& +\frac{1}{24} c_{1} c_{2}+\frac{1}{720}\left(-c_{1}^{4}+4 c_{1}^{2} c_{2}+3 c_{2}^{2}+c_{1} c_{3}-c_{4}\right)+\ldots
\end{aligned}
$$

Для выписанной выше точной последовательности векторных расслоений имеем

$$
\operatorname{td}(E)=\operatorname{td}\left(E^{\prime}\right) \operatorname{td}\left(E^{\prime \prime}\right) .
$$

Для полного класса Черна векторного расслоения $E$ ранга $r$ выполняется равенство

$$
\sum_{i=0}^{r}(-1)^{i} \operatorname{ch}\left(\Lambda^{i} E^{\vee}\right)=c_{r}(E) \operatorname{td}(E)^{-1} .
$$

Класс Тодда многообразия $M$ - это класс Тодда его касательного расслоения TM. Класс Тодда собственного отображсения $F: M \rightarrow N$ - это класс Тодда разности $F^{*} T N-T M$, т.е.

$$
\operatorname{td}(F)=\operatorname{td}\left(F^{*} T N-T M\right)=\frac{\operatorname{td} F^{*} T N}{\operatorname{td} T M} .
$$


3.1.3. Прямой образ когерентного пучка. Высший прямой образ $P_{!}(E)$ когерентного пучка $E$ на $M$ относительно отображения $P: M \rightarrow A$ - наиболее сложньй объект среди определенных в настоящем параграфе. Методы его вычисления зависят от соотношения размерностей многообразий $A$ и $M$. В частности, если размерности этих многообразий совпадают, то образ $P_{!}(E)$ совпадает с прямым образом $P_{*}(E)$. Напомним, что по определению прямы.м образом $P_{*} E$ пучка $E$ над $M$ относительно отображения $P: M \rightarrow A$ называется пучок, группа сечений которого над достаточно малым открытым множеством $U \subset A$ совпадает с ограничением пучка $E$ на $P^{-1}(U)$. Если $\operatorname{dim} M=\operatorname{dim} A+1$ (именно в этой ситуации мы и собираемся применять теорему ГРР), то

$$
P_{!}(E)=R^{0}\left(P_{*}(E)\right)-R^{1}\left(P_{*}(E)\right),
$$

где $R^{0}\left(P_{*}(E)\right)=P_{*}(E)$ - прямой образ пучка $E$, а $R^{1}\left(P_{*}(E)\right)$ - первый высший гротендиковский прямой образ, который можно вычислить с помошью двойственности Серра:

$$
R^{1}\left(P_{*}(E)\right)=\left(R^{0}\left(P_{*}\left(E^{\vee} \otimes \omega\right)\right)\right)^{\vee}=\left(P_{*}\left(E^{\vee} \otimes \omega\right)\right)^{\vee} ;
$$

здесь $\omega$ - относительный дуализирующий пучок.

3.2. Относительные классы Черна универсального расслоения. Вернемся теперь к коммутативному треугольнику (1.2) пространств и отображений. С этого момента мы будем пользоваться упрощенньми обозначениями, опуская индексы $g, n: X_{g, n}$ будет обозначаться через $X, f_{g, n}$ - через $f$ и т. д. Введем в пространстве $X$ (напомним, что $X$ - универсальная кривая над проективизированным пространством Гурвища) следуюшие классы когомологий, которые мы будем называть основным. чок отображения $p: X \rightarrow B$, т. е. единственное голоморфное линейное расслоение над $X$, слой которого над гладкой точкой слоя отображения $p$ совпадает с кокасательной прямой к этому слою. Первый класс Черна $c_{1}(\omega)$ является элементом группы вторых когомологий $H^{2}(X)$. Пусть П - дивизор полюсов универсального отображения $f$, т. е. множество точек в $X$, в которых ограничение этого отображения на слой имеет полюс. Напомним, что, поскольку каждое из пространств $X, Y$ и $B$ является пространством орбит действия группь $\mathbb{C}^{*}$, на каждом из них выделен класс когомологий $c_{1}(\mathcal{O}(1)) \in H^{2}$, который мы обозначаем соответственно через $\Psi_{X}, \Psi_{Y}$ и $\psi$. Введем также обозначение $\Sigma=c_{1}(\omega)+\Psi_{X}+2$ П для первого относительного класса Черна отображения $f$.

Помимо подмногообразий и классов коразмерности один нам также понадобится класс $\Delta \subset X$, представленный множеством особых точек слоев отображения $p$. Ранг нормального расслоения к $\Delta$ в $X$ равен двум. Локально это нормальное расслоение допускает естественное расшепление в прямую сумму двух линейных расслоений.

Действительно, каждая точка в $\Delta$ является точкой трансверсального пересечения двух ветвей кривой-слоя в этой точке, и касательные прямые к этим ветвям и задают расщепление нормального расслоения. (В случае рациональных кривых, $g=0$, это расщепление продолжается до глобального, поскольку двойная точка 
разбивает рациональную кривую на две компоненты связности, однако мы не будем ниже этим пользоваться.) Обозначим через $N$ первый класс Черна нормального расслоения к $\Delta \subset X$. Этот класс Черна можно считать оператором, который можно применять к $\Delta$. Его степени $N^{i}$ являются операторами того же вида. Второй класс Черна нормального расслоения можно отождествить с самим классом $\Delta$.

ЛЕмма 3.1. Основные классы удовлетворяют следующим соотношениям (в когомологиях $H^{*}(X)$ ):

$$
\Pi^{2}=-\Psi_{X} \Pi, \quad \Sigma \Pi=0, \quad \Pi \Delta=0, \quad \Sigma \Delta=\Psi_{X} \Delta .
$$

ДокАЗАТЕЛЬСТво. Равенство П $\Delta=0$ вытекает из того, что полюсы любой функции в пространстве Гурвица являются гладкими точками кривой, а значит, подмногообразия $\Delta$ и П не пересекаются. Аналогично, равенство $\Sigma \Pi=0$ служит алгебраическим выражением того факта, что все полюсы функции простые и ветвления над бесконечностью не происходит.

Дуализируюший пучок $\omega$ почти тривиален над $\Delta$ (его поднятие на двулистное накрытие над $\Delta$ тривиально), а в точках гиперповерхности П он изоморфен конормальному линейному расслоению к ней. Отсюда вытекают равенства $c_{1}(\omega) \Delta=0$ и $\Pi^{2}=-c_{1}(\omega) \Pi$. Подставляя в них $c_{1}(\omega)=\Sigma-\Psi_{X}-2 \Pi$, мы получаем соответственно

$$
\begin{aligned}
0 & =\left(\Sigma-\Psi_{X}-2 \Pi\right) \Delta=\Sigma \Delta-\Psi_{X} \Delta, \\
\Pi^{2} & =-\left(\Sigma-\Psi_{X}-2 \Pi\right) \Pi=\Psi_{X} \Pi+2 \Pi^{2},
\end{aligned}
$$

что доказывает равенства $\Sigma \Delta=\Psi_{X} \Delta$ и $\Pi^{2}=-\Psi_{X} \Pi$. Отметим, что последнее из них можно вывести и путем поднятия на $X$ равенства $\Pi_{Y}^{2}=-\Psi_{Y} \Pi_{Y}$, имеюшего место на $Y$ (см. следуюший п. 3.3).

СЛЕДСТВИЕ 3.2. Всякий многочлен от основных классов можно представить в виде

$$
P_{1}\left(\Psi_{X}\right) \Pi+P_{2}\left(\Psi_{X}, \Sigma\right)+P_{3}\left(\Psi_{X}, N, \Delta\right) \Delta,
$$

где $P_{1}, P_{2}, P_{3}$ - некоторые многочлены.

Основная цель настоящего параграфа состоит в доказательстве следуюшего утверждения, из которого мы затем выведем некоторые соотношения для прямых образов основных классов.

ТЕОРема 3.3. Полный относительный класс Черна отображения $f$ выражается формулой

$$
c(f)=\left(1+\Psi_{X}\right)\left(1+\frac{\Sigma}{1-\Sigma+\Psi_{X}}-\frac{\Delta}{1+N+\Delta}\right) .
$$

Отсюда получаем

СлЕДСТВИЕ 3.4. Если при ограничении на слои отображения р отображение $f$ может иметь особенности только типа $A_{k}$ или $I_{k, l}$, то остаточные многочлены $R_{\alpha_{1}, \ldots, \alpha_{c}}$ для мультимультиособенностей принадлежсат подкольиу в $H^{*}(X)$, порожденному основными классами $\Sigma, \Psi_{X}, \Delta, N$. 
Поскольку $p$ является композицией отображений $f$ и $q, p=f \circ q$, его полньй относительньй класс Черна является результатом деления поднятия полного относительного класса Черна отображения $q$ и полного относительного класса Черна отображения $f$ :

$$
c(f)=\frac{f^{*} c(q)}{c(p)} .
$$

Поэтому для его подсчета достаточно вычислить классы $c(p), c(q)$, а также поднятие последнего класса на $X$. Именно этим мы сейчас и займемся.

3.2.1. Класс $c(q)$ и его поднятие $f^{*} c(q)$. Тотальное пространство $Y$ расслоения $q$ является проективизацией векторного расслоения $E=\mathbb{C} \oplus \mathcal{O}(1)$ ранга два над $B$. Обозначим через $\mathcal{T}$ тавтологическое линейное расслоение над $Y$ и положим $\Pi_{Y}=$ $c_{1}\left(\mathcal{T}^{\vee}\right)=-c_{1}(\mathcal{T})$. Этот класс удовлетворяет соотношению $c_{2}\left(q^{*}(E) / \mathcal{T}\right)=0$, т.е.

$$
\Pi_{Y}^{2}+\Pi_{Y} \Psi_{Y}=0
$$

где $\Psi_{Y}=q^{*}(\psi)=c_{1}(\mathcal{O}(1))$ - характеристический класс действия группы $\mathbb{C}^{*}$. Полученное соотношение описывает кольцо когомологий пространства $Y$ как алгебру над $H^{*}(B)$, причем действие кольца $H^{*}(B)$ задается отображением $q^{*}$.

Прямые $\mathbb{C} \oplus\{0\}$ и $\{0\} \oplus \mathcal{O}(1)$ в слоях расслоения $E$ соответствуют точкам "нуль" и "бесконечность" в слоях проекции $q$. Эти точки образуют гиперповерхности с двойственными классами Пуанкаре

$$
c_{1}(\operatorname{Hom}(\mathcal{T}, \mathcal{O}(1)))=\Pi_{Y}+\Psi_{Y}, \quad c_{1}(\operatorname{Hom}(\mathcal{T}, \mathbb{C}))=\Pi_{Y}
$$

соответственно. Таким образом, $\Pi_{Y}$ можно интерпретировать как класс, двойственный к "множеству бесконечностей" в $Y$, а равенство (3.4) выражает тот факт, что множество нулей и множество бесконечностей не пересекаются.

Полный класс Черна проекции q обратен к полному классу Черна относительного касательного расслоения $\operatorname{ker}\left(q_{*}\right)$. Это расслоение дается изоморфизмом $\operatorname{ker}\left(q_{*}\right) \simeq \operatorname{Hom}\left(\mathcal{T}, q^{*}(E) / \mathcal{T}\right)$. Поэтому

$$
c(q)=\frac{1}{c\left(\operatorname{Hom}\left(\mathcal{T}, q^{*}(E) / \mathcal{T}\right)\right)}=\frac{1}{1+\Psi_{Y}+2 \Pi_{Y}} .
$$

Применяя $f^{*}$, получаем $f^{*}\left(\Psi_{Y}\right)=\Psi_{X}$ и $f^{*}\left(\Pi_{Y}\right)=\Pi$, откуда, наконец, имеем

$$
f^{*}(c(q))=\frac{1}{1+\Psi_{X}+2 \Pi}
$$

3.2.2. Полный класс Черна отображения $p$. Вычисление полного класса Черна отображения $p$ несколько сложнее. Его можно подсчитать, воспользовавшись точной последовательностью

$$
0 \longrightarrow p^{*} T^{\vee} B \stackrel{p^{\vee}}{\longrightarrow} T^{\vee} X \longrightarrow \omega \longrightarrow \mathcal{O}_{\Delta} \longrightarrow 0,
$$


где, напомним, $\omega$ обозначает относительный дуализирующий пучок отображения $p$. Подмногообразие $\Delta \subset X-$ это в точности множество тех точек, где коранг отображения $p^{*}$ равен не единице, а двум. Структурный пучок $\mathcal{O}_{\Delta}$ подмногообразия $\Delta$ измеряет разность между относительным дуализирующим пучком $\omega$ и пучком относительных дифференциалов.

Применяя к выписанной выше точной последовательности формулу Уитни, мы получаем

$$
c\left(p^{\vee}\right)=1-c_{1}(p)+c_{2}(p)-\ldots=c\left(p^{*} T^{\vee} B-T^{\vee} X\right)=\frac{c\left(\mathcal{O}_{\Delta}\right)}{c(\omega)}=\frac{c\left(\mathcal{O}_{\Delta}\right)}{1+c_{1}(\omega)} .
$$

Для вычисления класса Черна $c\left(\mathcal{O}_{\Delta}\right)$ воспользуемся проективной резольвентой Кошуля (3.2). Еще раз применяя формулу Уитни, имеем

$$
c\left(\mathcal{O}_{\Delta}\right)=\frac{1-N}{1-N+\Delta}=1-\frac{\Delta}{1-N+\Delta} .
$$

Эту формулу можно вывести, применив формулу ГРР к вложению $\Delta \hookrightarrow X$. Подставляя ее в выражение для $c\left(p^{\vee}\right)$, получаем

$$
c(p)=\frac{1}{1-c_{1}(\omega)}\left(1-\frac{\Delta}{1+N+\Delta}\right) .
$$

Теперь можно подсчитать полный класс Черна отображения $f$. Применяя соотношения из леммы 3.1 , имеем

$$
\begin{aligned}
c(f) & =\frac{c(p)}{f^{*}(c(q))}=\frac{1+\Psi_{X}+2 \Pi}{1-c_{1}(\omega)}\left(1-\frac{\Delta}{1+N+\Delta}\right) \\
& =\frac{1+\Psi_{X}+2 \Pi}{1-\Sigma+\Psi_{X}+2 \Pi}-\frac{1+\Psi_{X}}{1+N+\Delta} \Delta \\
& =\left(\frac{1+\Psi_{X}}{1-\Sigma+\Psi_{X}}-\frac{1+\Psi_{X}}{1+N+\Delta}\right)+\left(\frac{1+\Psi_{X}+2 \Pi}{1-\Sigma+\Psi_{X}+2 \Pi}-\frac{1+\Psi_{X}}{1-\Sigma+\Psi_{X}}\right) .
\end{aligned}
$$

Первое слагаемое совпадает с правой частью равенства (3.3); после приведения к общему знаменателю второго слагаемого его числитель оказывается равным

$$
\left(1+\Psi_{X}+2 \Pi\right)\left(1-\Sigma+\Psi_{X}\right)-\left(1+\Psi_{X}\right)\left(1-\Sigma+\Psi_{X}+2 \Pi\right)=-2 \Pi \Sigma=0 .
$$

Теорема 3.3 доказана.

3.3. Гомоморфизмы прямого образа. Для того чтобы иметь возможность применять универсальные формулы к классам мультимультиособенностей, необходимо описание гомоморфизмов прямого образа $f_{*}, p_{*}, q_{*}$. Следующая теорема утверждает, что гомоморфизм $p_{*}$ определяет остальные гомоморфизмы.

ТЕОРема 3.5. Для любого набора классов когомологий $h_{1}, \ldots, h_{s}$ на $X$, удовлетворяющих условию $h_{i} \Pi=0, i=1, \ldots, s$, справедливо равенство

$$
q_{*}\left(f_{*}\left(h_{1}\right) \ldots f_{*}\left(h_{s}\right)\right)=\psi^{s-1} p_{*}\left(h_{1}\right) \ldots p_{*}\left(h_{s}\right) .
$$

Геометрически условие $h_{i} \Pi=0$ означает, что класс $h_{i}$ можно представить циклом, не пересекаюшимся с дивизором полюсов П. Для остаточных многочленов мультимультиособенностей оно выполняется. 
ДокАЗАТЕЛЬСТво. Поскольку $q$-проективизация векторного расслоения, прямой образ $f_{*}(h)$ произвольного класса $h \in H^{*}(X)$ можно представить в виде

$$
f_{*}(h)=q^{*}(a)+q^{*}(b) \Pi_{Y}
$$

для некоторых классов $a$ и $b$ на $B$. Класс $b$ можно вычислить, применяя $q_{*}$ к обеим частям этого равенства. Из равенства $q_{*} \Pi_{Y}=1$ и формулы проекции мы заключаем, что

$$
b=q_{*}\left(f_{*}(h)\right)=p_{*}(h) .
$$

Класс $a$ вычисляется аналогичным образом: умножая обе части выписанного вьше уравнения на $\Pi_{Y}+\Psi_{Y}$, получаем

$$
f_{*}\left(\left(\Psi_{X}+\Pi\right) h\right)=q^{*}(a)\left(\Psi_{Y}+\Pi_{Y}\right)+q^{*}(b)\left(\Psi_{Y}+\Pi_{Y}\right) \Pi_{Y}=q^{*}(\psi a)+q^{*}(a) \Pi_{Y},
$$

и, применяя $q_{*}$, находим $a=p_{*}\left(\left(\Psi_{X}+\Pi\right) h\right)$. Если класс $h$ удовлетворяет предположению теоремы $h \Pi=0$, то ответ упрошается:

$$
f_{*}(h)=q^{*}\left(p_{*}(h)\right)\left(\Psi_{Y}+\Pi_{Y}\right) .
$$

Поэтому в предположениях теоремы справедливо

$$
f_{*}\left(h_{1}\right) \ldots f_{*}\left(h_{s}\right)=\left(\Psi_{Y}+\Pi_{Y}\right)^{s} \prod_{i=1}^{s} q^{*}\left(p_{*}\left(h_{i}\right)\right)=\left(\Psi_{Y}^{s}+\Psi_{Y}^{s-1} \Pi_{Y}\right) \prod_{i=1}^{s} q^{*}\left(p_{*}\left(h_{i}\right)\right)
$$

Применяя $q_{*}$, мы с помощью формулы проекции получаем требуемое равенство.

Таким образом, для завершения вычисления классов когомологий стратов в пространстве Гурвища $P \overline{\mathcal{H}}_{g, n}$ мы должны уметь вычислять прямой образ $p_{*}(R)$ всякого остаточного многочлена, представленного в виде

$$
R=P_{1}\left(\Psi_{X}, \Sigma\right)+P_{2}\left(\Psi_{X}, N, \Delta\right) \Delta
$$

Положим

$$
\xi_{k}=p_{*}\left(\Sigma^{k+1}\right), \quad \delta_{k, \ell}=p_{*}\left(N^{k} \Delta^{\ell+1}\right) .
$$

Между классами $\xi_{k}$ и $\delta_{k, \ell}$ нет никаких универсальных соотношений, справедливых при всех значениях $g$ и $n$. Разумеется, для любых конкретных $g$ и $n$ размерность пространства когомологий $H^{*}\left(P \overline{\mathcal{H}}_{g, n}\right)$ конечна, поэтому для фиксированных $g$ и $n$ такие соотношения сушествуют. Некоторые из них обсуждаются в следующем п. 3.4. 
3.4. Соотношения на классы когомологий в $B$. Применим формулу ГРР к проекции $p: X \rightarrow B$ и к относительному дуализирующему пучку $\omega$. Будем действовать аналогично работе [18], где то же самое реализовано для проекции универсальной кривой на пространство модулей кривых. Имеем

$$
\operatorname{ch}\left(p_{!} \omega\right)=p_{*}\left(\operatorname{ch}(\omega) \operatorname{td}(p)^{-1}\right)
$$

Пучок в левой части $p_{!} \omega=R^{0} p_{*} \omega-R^{1} p_{*} \omega$ выражается через расслоение Ходжа $\Lambda=\Lambda_{g, n}$ над $B=P \overline{\mathcal{H}}_{g, n}$ (напомним, что слой расслоения Ходжа над точкой $(C ; f)$ - это пространство голоморфных 1-форм на $C$; тем самым ранг этого расслоения равен $g$ ): $R^{0} p_{*} \omega=p_{*} \omega=\Lambda$ и, по двойственности Серра, $R^{1} p_{*} \omega=p_{*} \mathbb{C}=\mathbb{C}$. Классы в правой части формулы были вычислены выше. Воспользовавшись тем, что $c_{1}(\omega) \Delta=0$, получаем

$$
\begin{aligned}
\operatorname{ch}(\omega) \operatorname{td}^{-1}(p) & =\operatorname{ch}(\omega) \operatorname{td}\left(\omega^{\vee}-\mathcal{O}_{\Delta}^{\vee}\right)=e^{c_{1}(\omega)} \frac{-c_{1}(\omega)}{1-e^{c_{1}(\omega)}} \operatorname{td}\left(-\mathcal{O}_{\Delta}^{\vee}\right) \\
& =\frac{c_{1}(\omega)}{1-e^{-c_{1}(\omega)}}+\operatorname{td}\left(-\mathcal{O}_{\Delta}^{\vee}\right),
\end{aligned}
$$

где класс Тодда (виртуального) пучка $-\mathcal{O}_{\Delta}^{\vee}$ определяется его классом Черна

$$
c\left(-\mathcal{O}_{\Delta}^{\vee}\right)=\frac{1+N+\Delta}{1+N}=1+\frac{\Delta}{1+N} .
$$

Подставляя результат в формулу ГРР, получаем

$$
\begin{aligned}
(g-1) & +\operatorname{ch}_{1}(\Lambda)+\operatorname{ch}_{2}(\Lambda)+\operatorname{ch}_{3}(\Lambda)+\operatorname{ch}_{4}(\Lambda)+\operatorname{ch}_{5}(\Lambda)+\ldots \\
= & \frac{1}{2} p_{*}\left(c_{1}(\omega)\right)+\frac{1}{12} p_{*}\left(c_{1}^{2}(\omega)+\Delta\right)-\frac{1}{720} p_{*}\left(c_{1}^{4}(\omega)+\left(N^{2}-3 \Delta\right) \Delta\right) \\
& +\frac{1}{30240} p_{*}\left(c_{1}^{6}(\omega)+\left(N^{4}-5 N^{2} \Delta+5 \Delta^{2}\right) \Delta\right)+\ldots
\end{aligned}
$$

Это соотношение показывает, что однородные компоненты характера Черна (а значит, и классы Черна) расслоения Ходжа выражаются через прямые образы основных классов.

Отметим, что в правой части равенства (3.6) отсутствуют члены положительных четных степеней. Отсюда вытекает, что четная часть характера $\operatorname{ch}(\Lambda)$ тривиальна, т. е. $c\left(\Lambda+\Lambda^{\vee}\right)=1$. В случае $g=0$ само расслоение Ходжа тривиально, а значит, и левая часть равенства (3.6) обрашается в нуль при положительных степенях. Отсюда вытекают нетривиальные тождества для прямых образов основных классов:

$$
p_{*}\left(c_{1}^{2}(\omega)+\Delta\right)=0, \quad p_{*}\left(c_{1}^{4}(\omega)+\left(N^{2}-3 \Delta\right) \Delta\right)=0, \quad \ldots
$$




\section{§4. Применение универсальных многочленов к изучению стратификации пространств Гурвица}

Знание остаточных многочленов для особенностей, мультиособенностей и мультимультиособенностей позволяет подсчитать когомологические классы стратов в пространствах Гурвица. Полные такие формулы должны учитывать и вклад неизолированных особенностей, который мы пока не смогли подсчитать. Именно поэтому мы приводим универсальные выражения для стратов только в случае рациональных функций и только для малых коразмерностей.

4.1. Универсальные выражения для стратов. Ниже приведены выражения для стратов коразмерности один и два по модулю классов с носителями на стратах неизолированных особенностей в терминах прямых образов основных классов, полученные на основе вычисленных остаточных классов (мы используем обозначения $\left.\xi_{k}=p_{*}\left(\Sigma^{k+1}\right), \xi_{0}=2 n-2+2 g, \delta_{k, l}=p_{*}\left(N^{k} \Delta^{l+1}\right)\right)$ :

$$
\begin{aligned}
\sigma_{2^{1}}= & -\psi \xi_{0}+2 \xi_{1}-\delta_{0,0} \\
\sigma_{1^{2}}= & \frac{1}{2} \psi \xi_{0}\left(\xi_{0}+2\right)-3 \xi_{1}+\delta_{0,0} \\
\sigma_{3^{1}}= & 2 \xi_{0} \psi^{2}-7 \xi_{1} \psi-5 \delta_{0,0} \psi+6 \xi_{2}+2 \delta_{1,0}, \\
\sigma_{1^{1} 2^{1}}= & -\xi_{0}\left(\xi_{0}+6\right) \psi^{2}+2\left(\xi_{0}+12\right) \xi_{1} \psi+\left(18-\xi_{0}\right) \delta_{0,0} \psi-24 \xi_{2}-6 \delta_{1,0}, \\
\sigma_{1^{3}}= & \frac{1}{6} \xi_{0}\left(\xi_{0}^{2}+6 \xi_{0}+24\right) \psi^{2}-\frac{1}{3}\left(9 \xi_{0}+52\right) \xi_{1} \psi \\
& +\frac{1}{3}\left(3 \xi_{0}-40\right) \delta_{0,0} \psi+20 \xi_{2}+4 \delta_{1,0}, \\
& +\left(\xi_{0}+7\right) \delta_{0,0} \psi-2 \xi_{1} \delta_{0,0}-\frac{5}{2} \delta_{1,0}, \\
\sigma_{2^{1}, 2^{1}}= & \frac{1}{2}\left(\xi_{0}-6\right) \xi_{0} \psi^{2}-\left(2 \xi_{0}-11\right) \xi_{1} \psi+\frac{1}{2} \delta_{0,0}^{2}+2 \xi_{1}^{2}-10 \xi_{2} \\
& +\frac{1}{2}\left(\xi_{0}^{2}+42\right) \delta_{0,0} \psi+5 \xi_{1} \delta_{0,0}+6 \delta_{1,0}, \\
\sigma_{2^{1}, 1^{2}}= & \frac{1}{2} \xi_{0}\left(\xi_{0}^{2}-2 \xi_{0}-12\right) \psi^{2}+\left(\xi_{0}^{2}+\xi_{0}-27\right) \xi_{1} \psi-\delta_{0,0}^{2}-6 \xi_{1}^{2}+30 \xi_{2} \\
\sigma_{1^{2}, 1^{2}}= & \frac{1}{8}\left(\xi_{0}-4\right) \xi_{0}\left(\xi_{0}^{2}+4 \xi_{0}+6\right) \psi^{2}-\frac{3}{4}\left(2 \xi_{0}^{2}-4 \xi_{0}-21\right) \xi_{1} \psi+\frac{1}{2} \delta_{0,0}^{2} \\
& +\frac{45}{2} \xi_{2}+\frac{1}{4}\left(2 \xi_{0}^{2}-4 \xi_{0}+57\right) \delta_{0,0} \psi-3 \xi_{1} \delta_{0,0}-\frac{7}{2} \delta_{1,0} .
\end{aligned}
$$

Теперь приведем выражения для тех же самых стратов в случае $g=0$, упрощенные с учетом тождеств

$$
\xi_{0}=2 n-2, \quad \xi_{1}=4(n-1) \psi-\delta_{0,0}
$$

из п. 3.4 (отметим, что в случае $g=0$ коразмерность множества неизолированных особенностей в $B$ равна трем, поэтому они не дают вклада в выписанные выражения):

$$
\begin{aligned}
& \sigma_{2^{1}}=6(n-1) \psi-3 \delta_{0,0}, \\
& \sigma_{1^{2}}=2(n-6)(n-1) \psi+4 \delta_{0,0},
\end{aligned}
$$




$$
\begin{aligned}
\sigma_{3^{1}} & =-24(n-1) \psi^{2}+2 \delta_{0,0} \psi+6 \xi_{2}+2 \delta_{1,0}, \\
\sigma_{1^{1} 2^{1}} & =12(n-1)(n+6) \psi^{2}-6 n \delta_{0,0} \psi-24 \xi_{2}-6 \delta_{1,0}, \\
\sigma_{1^{3}}= & \frac{4}{3}(n-1)\left(n^{2}-17 n-30\right) \psi^{2}+4(2 n-1) \delta_{0,0} \psi+20 \xi_{2}+4 \delta_{1,0}, \\
\sigma_{2^{1}, 2^{1}}= & 2(n-1)(9 n+10) \psi^{2}-2(9 n-7) \delta_{0,0} \psi+\frac{9}{2} \delta_{0,0}^{2}-10 \xi_{2}-\frac{5}{2} \delta_{1,0}, \\
\sigma_{2^{1}, 1^{2}}= & 12(n-9)(n-1) n \psi^{2}-6\left(n^{2}-13 n+11\right) \delta_{0,0} \psi-12 \delta_{0,0}^{2}+30 \xi_{2}+6 \delta_{1,0}, \\
\sigma_{1^{2}, 1^{2}}= & (n-1)\left(2 n^{3}-30 n^{2}+145 n-60\right) \psi^{2}+\frac{1}{2}\left(16 n^{2}-144 n+125\right) \delta_{0,0} \psi \\
& +8 \delta_{0,0}^{2}-\frac{45}{2} \xi_{2}-\frac{7}{2} \delta_{1,0} .
\end{aligned}
$$

4.2. Степени стратов рода нуль. В случае $g=0$ компактифицированное пространство Гурвица $P \overline{\mathcal{H}}_{0, n}$ расслоено над компактифицированным пространством модулей $\overline{\mathcal{M}}_{0, n}$ рациональных кривых с отмеченными точками. Слоем этого расслоения служит проективное пространство $P E$, где $E=L_{1}^{\vee} \oplus \cdots \oplus L_{n}^{\vee}-$ сумма Уитни касательных прямых к кривой в ее отмеченных точках. Отсюда вытекает, что когомологии пространства $P \overline{\mathcal{H}}_{0, n}$, рассматриваемые как алгебра над $H^{*}\left(\overline{\mathcal{M}}_{0, n}\right)$, порождены классом $\psi=c_{1}(\mathcal{O}(1))$, подчиняющимся соотношению

$$
\psi^{n}+c_{1}(E) \psi^{n-1}+\cdots+c_{n}(E)=0
$$

(cp. [16]). В частности, всякий класс $\alpha$ в $H^{2 d}\left(P \overline{\mathcal{H}}_{0, n}\right)$ можно представить в виде

$$
\alpha=\pi^{*}\left(\eta_{d}\right)+\pi^{*}\left(\eta_{d-1}\right) \psi+\pi^{*}\left(\eta_{d-2}\right) \psi^{2}+\ldots,
$$

где $\pi: P \overline{\mathcal{H}}_{0, n} \rightarrow \overline{\mathcal{M}}_{0, n}$ - естественная проекция, а классы $\eta_{i}$ лежат в когомологиях пространства $\overline{\mathcal{M}}_{0, n}$. Из хорошо известного соотношения $\pi_{*} \psi^{s}=c_{s-n+1}(-E)$ (см., например, [3]) вытекает, что степень любого класса, представленного в таком виде, можно вычислить, воспользовавшись соотношением

$$
\operatorname{deg} \alpha=\int_{P \overline{\mathcal{H}}_{0, n}} \frac{\pi^{*}\left(\eta_{d}\right)+\pi^{*}\left(\eta_{d-1}\right) \psi+\ldots}{1-\psi}=\int_{\overline{\mathcal{M}}_{0, n}} \frac{\eta_{d}+\eta_{d-1}+\eta_{d-2}+\ldots}{c(E)} .
$$

Таким образом, вычисление степени сводится к вычислению некоторых интегралов по пространствам модулей рациональных кривых с отмеченными точками, значения которых хорошо известны (см., например, [2] или [14]). Ниже мы приводим результаты вычислений степеней прямых образов основных классов вплоть до коразмерности два:

$$
\begin{aligned}
\operatorname{deg}(1) & =n^{n-3}, \\
\operatorname{deg}\left(\delta_{0,0}\right) & =\frac{1}{2}(n-1)(n+6) n^{n-4}, \\
\operatorname{deg}\left(\xi_{2}\right) & =\frac{1}{3}(n-1)\left(17 n^{2}-28 n+12\right) n^{n-5}, \\
\operatorname{deg}\left(\delta_{1,0}\right) & =-\frac{1}{6}(n-1)\left(n^{2}+10 n-120\right) n^{n-5}, \\
\operatorname{deg}\left(\delta_{0,0}^{2}\right) & =\frac{1}{12}(n-1)\left(3 n^{3}+31 n^{2}+82 n-120\right) n^{n-5} .
\end{aligned}
$$


Подставляя эти равенства в вычисленные разложения стратов, мы получаем следуюшие формулы для их степеней:

$$
\begin{aligned}
\operatorname{deg}\left(\sigma_{2^{1}}\right) & =\frac{9}{2}(n-2)(n-1) n^{n-4}, \\
\operatorname{deg}\left(\sigma_{1^{2}}\right) & =2(n-3)(n-2)(n-1) n^{n-4}, \\
\operatorname{deg}\left(\sigma_{3^{1}}\right) & =\frac{32}{3}(n-3)(n-2)(n-1) n^{n-5}, \\
\operatorname{deg}\left(\sigma_{1^{1} 2^{1}}\right) & =9(n-4)(n-3)(n-2)(n-1) n^{n-5}, \\
\operatorname{deg}\left(\sigma_{1^{3}}\right) & =\frac{4}{3}(n-5)(n-4)(n-3)(n-2)(n-1) n^{n-5}, \\
\operatorname{deg}\left(\sigma_{2^{1}, 2^{1}}\right) & =\frac{3}{8}(n-2)(n-1)\left(27 n^{2}-137 n+180\right) n^{n-5}, \\
\operatorname{deg}\left(\sigma_{2^{1}, 1^{2}}\right) & =3(n-3)(n-2)(n-1)\left(3 n^{2}-15 n+20\right) n^{n-5}, \\
\operatorname{deg}\left(\sigma_{1^{2}, 1^{2}}\right) & =(n-3)(n-2)(n-1)\left(2 n^{3}-16 n^{2}+43 n-40\right) n^{n-5} .
\end{aligned}
$$

Из теоремы 1.1 вытекают следующие формулы для чисел Гурвища:

$$
\begin{gathered}
h_{2^{1}}=\frac{9}{2} \frac{(2 n-4) !}{(n-3) !} n^{n-5}, \\
h_{1^{2}}=2 \frac{(2 n-4) !}{(n-4) !} n^{n-5}, \\
h_{3^{1}}=\frac{32}{3} \frac{(2 n-5) !}{(n-4) !} n^{n-6}, \\
h_{1^{1} 2^{1}}=9 \frac{(2 n-5) !}{(n-5) !} n^{n-6}, \\
h_{1^{3}}=\frac{4}{3} \frac{(2 n-5) !}{(n-6) !} n^{n-6}, \\
h_{2^{1}, 2^{1}}=\frac{3}{4}\left(27 n^{2}-137 n+180\right) \frac{(2 n-6) !}{(n-3) !} n^{n-6}, \\
h_{2^{1}, 1^{2}}=3\left(3 n^{2}-15 n+20\right) \frac{(2 n-6) !}{(n-4) !} n^{n-6}, \\
h_{1^{2}, 1^{2}}=2\left(2 n^{3}-16 n^{2}+43 n-40\right) \frac{(2 n-6) !}{(n-4) !} n^{n-6} ;
\end{gathered}
$$

здесь первая группа формул относится к случаю одного вырожденного ветвления, эти формулы представляют собой частные случаи формулы Гурвица.

Другой подход к вычислению степеней стратов состоит в непосредственном применении формулы Гурвища (или, для произвольного рода, формулы из [2], которая, однако, менее явная) для чисел Гурвица мультиособенностей. Теорема 1.1 позволяет нам определить степень каждого страта вида $\sigma_{\alpha}$. В рациональном случае $g=0$, зная эти разложения стратов по прямым образам основных, мы можем вычислить степени по крайней мере некоторых из этих прямых образов. В нашем 
случае легко вычисляются степени $\operatorname{deg}(1), \operatorname{deg}\left(\delta_{0,0}\right), \operatorname{deg}\left(\delta_{1,0}\right), \operatorname{deg}\left(\xi_{2}\right)$. Отметим, однако, что знание этих степеней недостаточно для подсчета степеней стратов мультимультиособенностей: в их разложение входит класс $\delta_{0,0}^{2}$. Его степень можно подсчитать, например, воспользовавшись степенью страта $\sigma_{2^{1}, 2^{1}}$, которая известна из работы [22].

4.3. О неизолированных особенностях. Хотя мы не имеем еще полной картины происходящего при включении в рассмотрение неизолированных особенностей, следует все-таки сказать несколько слов о них. Рассмотрим первый нетривиальный вклад неизолированных особенностей в случае рациональных функций, т. е. случай классов коразмерности три. Обозначим через $\sigma_{\alpha_{1}, \alpha_{2}, \ldots}^{\exp }$ ожидаемые разложения для соответствуюших классов, полученные по универсальным формулам в предположении отсутствия особенностей типов, отличных от $A_{k}$ и $I_{k, l}$. Они отличаются от правильных разложений классов $\sigma_{\alpha_{1}, \alpha_{2}, \ldots}$ членами с носителями на множестве $I_{\infty} \subset B$ функций с неизолированньми особенностями. Поскольку поправочные члены имеют ту же размерность, что и подмногообразие $I_{\infty}$, они должны быть пропорциональны фундаментальному классу этого подмногообразия с некоторым постоянным коэффициентом пропорциональности. Естественно предполагать, что этот коэффициент универсален, т. е. он не зависит ни от $n$, ни от выбора неприводимой компоненты подмногообразия $I_{\infty}$. Численным подтверждением сделанного предположения служит то "неожиданное" наблюдение, что степень разности

$$
\operatorname{deg}\left(\sigma_{\alpha_{1}, \alpha_{2}, \ldots}-\sigma_{\alpha_{1}, \alpha_{2}, \ldots}^{\exp }\right)
$$

пропорциональна степени страта $I_{\infty}$,

$$
\operatorname{deg}\left(I_{\infty}\right)=\frac{1}{8}(n-1)\left(n^{3}+11 n^{2}+34 n-120\right) n^{n-5},
$$

с цельп коэффициентом. Степени стратов $\sigma_{\alpha_{1}, \alpha_{2}, \ldots}^{\exp }$ вычисляются методами, описанными в настоящей статье, а истинную степень страта $\sigma_{\alpha_{1}, \alpha_{2}, \ldots}$ можно вывести из формулы Гурвица. Приведем гипотетические вклады неизолированных особенностей в некоторые страты коразмерности три:

$$
\begin{aligned}
\sigma_{4^{1}} & =\sigma_{4^{1}}^{\exp }+5 I_{\infty}, \\
\sigma_{1^{1} 3^{1}} & =\sigma_{1^{1} 3^{1}}^{\exp }-16 I_{\infty}, \\
\sigma_{2^{2}} & =\sigma_{2^{2}}^{\exp }-9 I_{\infty}, \\
\sigma_{1^{2} 2^{1}} & =\sigma_{1^{2} 2^{1}}^{\exp }+36 I_{\infty}, \\
\sigma_{1^{4}} & =\sigma_{1^{4}}^{\exp }-16 I_{\infty} .
\end{aligned}
$$

\section{Список литературы}

1. Ekedahl T., Lando S. K., Shapiro M., Vainshtein A. On Hurwitz numbers and Hodge integrals // C. R. Acad. Sci. Paris. Sér. I. Math. 1999. V. 328. P. 1175-1180.

2. Ekedahl T, Lando S. K., Shapiro M., Vainshtein A. Hurwitz numbers and intersections on moduli spaces of curves // Invent. math. 2001. V. 146. P. 297-327.

3. Фултон В. Теория пересечений. М.: Мир, 1994. 
4. Giusti M. Classification des singularités isolées simples d'intersections complètes // Proceedings of Symposia in Pure Mathematics. 1983. V. 40. Part 1. P. 457-494.

5. Горюнов В. В. Особенности проектирований полных пересечений // Итоги науки и техники. Современные проблемы математики. Т. 22. М.: ВИНИТИ, 1983. С. 167-206.

6. Goulden I. P., Jackson D. M. Transitive factorisation into transpositions and holomorphic mappings on the sphere // Proc. Amer. Math. Soc. 1997. V. 125. № 1. P. 51-60.

7. Harris J., Mumford D. On the Kodaira dimension of the moduli space of curves // Invent. Math. 1982. V. 67. № 1. P. 23-88.

8. Hurwitz A. Über Riemann'sche Flächen mit gegebenen Verzweigungpunkten // Math. Ann. 1891. V. 39. P. 1-61.

9. Hurwitz A. Über die Anzal der Riemann'sche Flächen mit gegebenen Verzweigungpunkten // Math. Ann. 1902. V. 55. P. 51-60.

10. Казарян M. Э. Мультиособенности, кобордизмы и перечислительная геометрия // УМН. 2003. T. 58. № 4. C. $665-724$.

11. Kazaryan M. E. Morin maps and their characteristic classes. http://www.mi.ras.ru/kazaryan.

12. Kazaryan M.E. Classifying spaces of singularities and Thom polynomials // NATO Sci. Ser. II. Math. Phys. Chem. V.21. New developments in singularity theory. Dordrecht: Kluwer Acad. Publ., 2001. P. 117-134.

13. Казарян $M . Э$. Относительная теория Морса одномерных слоений и циклические гомологии // Функцион. анализ и его прилож. 1997. Т. 31. № 1. С. 20-31.

14. Ландо C.K. Разветвленные накрытия двумерной сферы и теория пересечений в пространствах мероморфных функций на алгебраических кривых // УМН. 2002. Т. 57. № 3. C. $463-533$.

15. Ландо С. К., Звонкин Д. А. О кратностях отображения Ляшко-Лойенги на стратах дискриминанта // Функцион. анализ и его прилож. 1999. Т. 33. № 3. С. 178-188.

16. Lando S. K., Zvonkine D. Counting ramified coverings and intersection theory on spaces of rational functions. I. math.AG/0303218.

17. Manin Yu., Zograf P. Invertible cohomological field theories and Weil-Petersson volumes // Ann. Inst. Fourier (Grenoble). 2000. V. 50. № 2. P. 519-535.

18. Mumford D. Towards an enumerative geometry on the moduli spaces of curves // Progress in Math. V. 36. Boston: Birkhüaser, 1983. P. 271-328.

19. Natanzon S. M., Turaev V. A compactification of the Hurwitz space // Topology. 1999. V. 38. P. 889-914.

20. Том P. Некоторые свойства "в целом" дифференцируемых многообразий // Расслоенные пространства и их приложения. М.: ИЛ, 1958. С. 293-351.

21. Zvonkine D. Multiplicities of the Lyashko-Looijenga map on its strata // C. R. Acad. Sci. 1997. V. 324. Sér. I. P. 1349-1353.

22. Zvonkine D. Counting ramified coverings and intersection theory on Hurwitz spaces. II (Local structures of Hurwitz spaces and combinatorial results). Preprint, math.AG/0304251.

Институт математики им. В. А. Стеклова РАН и

Независимый Московский университет,

Институт системных исследований РАН и

Независимый Московский университет
Поступило в редакцию

01.04 .2004 